\title{
Modeling the Evolution of Degree Correlation in Scale-Free Topology Generators
}

\author{
Xiaoming Wang, Xiliang Liu, and Dmitri Loguinov
}

\begin{abstract}
In this paper, we examine the asymptotic behavior of degree correlation (i.e., the joint degree distribution of adjacent nodes) in several scale-free topology generators GED [14], PLRG [1], GLP [11], BA [4], AB [2]. We present a unifying analytical framework that allows tractable analysis of degree correlation in all studied models and derive asymptotic formulas of two degree correlation metrics - assortativity and clustering. Our results indicate that all studied generators become uncorrelated as graph size increases, which is inconsistent with time-invariance of these metrics in real networks such as the Internet [37], [49], [51]. Since the class of degree-based generators is incapable of reproducing evolving characteristics of the Internet, we study three other models that evolve graphs using different rules than preference of degree (e.g., based on random walks [51], optimization [18], and geometry [24]) and show using simulations that these models are much more viable alternatives for replicating the complex structure of Internet-like graphs.
\end{abstract}

\section{INTRODUCTION}

Recent research suggests that many graphs found in the real world (such as social relationships, scientific collaborations, Internet autonomous system connectivity, web-page linkage, telephone call logs, various molecular structures, etc.) exhibit drastically different characteristics from those of classical Erdös-Rényi random graphs [17]. Besides the well-known heavy-tailed distribution of node degree [15], [19], real-world graphs demonstrate a strong correlation among the degree of adjacent nodes (e.g., large-degree nodes are more likely to be paired with small-degree nodes, or triangle formation is more likely between large-degree nodes). A significant research effort is currently under way to better understand the evolution of complex networks and design generators that can reproduce graph theoretic metrics found in these structures [1], [2], [4], [11], [13], [18], [23], [24], [28], [48], [51], [53].

Recent studies [29], [42] demonstrate that degree correlation (formally defined below) is sufficient for characterizing the structure of a random graph, where correlation among up to three neighboring nodes is enough to capture many commonly used graph properties. In fact, the authors of [29] propose a generator that randomly rewires links until it can match the desired degree correlation. The resulting graphs are then shown to replicate such global properties as coreness, spectrum, distance distribution, and betweenness of many existing topology generators. However, this approach is very

A shorter version of the paper appeared in IEEE INFOCOM 2008.

$X$. Wang and D. Loguinov are with the Department of Computer Science, Texas A\&M University, College Station, TX 77843 USA (e-mail: xmwang@cs.tamu.edu; dmitri@cs.tamu.edu).

X. Liu is with The City University of New York, New York, NY 10016 USA (e-mail: liuxiliang@gmail.com). computationally intensive and requires the knowledge of the joint degree distribution of neighbors in the target graph, which may be difficult to obtain in practice. Furthermore, the bruteforce approach of [29] does not easily allow the graph to evolve and does not capture any qualitative characteristics of the system it creates.

To understand the fundamental properties of degree correlation in existing topology generators and how well they match those of existing networks, we propose a new analytical framework for modeling two- and three-node correlation in powerlaw random graphs with a specific focus on the evolution of the system. It is well-known that static properties of graphs (i.e., those observed at a fixed time $t$ ) can be captured with sufficient accuracy by many existing generators. However, a more interesting question [51] has recently emerged as to whether these generators can replicate the behavior of real graphs as time evolves. Specifically, for a fixed set of initial parameters, [51] shows that the trend of clustering coefficients of many evolving degree-based generators exhibits a significant discrepancy with that of the Internet as observed in [37], [49]. As clustering coefficients are a special case of three-node correlation, our proposed framework allows to understand what causes this discrepancy and whether it can be overcome within the studied class of topology generators.

The class of models we study in this work is called degreebased since link formation between individual nodes depends only on the current degree of potential neighbors, or equivalently, weights assigned to them. Many traditional generators such as GED [14], PLRG [1], GLP [11], BA [4], and AB [2] fall into this category. On the other hand, generators that implement neighbor selection based on additional information (besides node degree) constitute a completely different class of link-based models (e.g., WIT [51], HOT [18], and SWT [24]), which are not modeled in this paper, but briefly studied in simulations (see below).

\section{A. Main Results}

We first study GED as a foundation for understanding correlation in degree-based random graphs. We derive the asymptotic decay rate of the expected assortativity coefficient $r(G)$, which captures two-node correlation in the system, and the expected clustering coefficient $\gamma(G)$, which captures threenode correlation, as graph size $n \rightarrow \infty$. Specifically, our analysis demonstrates that:

$$
E[r(G)]= \begin{cases}\Theta\left(-n^{1-\alpha}\right) & 1<\alpha<2 \\ \Theta\left(-n^{-1} \log ^{2} n\right) & \alpha=2 \\ 0 & \alpha>2\end{cases}
$$


and

$$
E[\gamma(G)]=\left\{\begin{array}{ll}
\Theta\left(n^{1-\alpha} \log n\right) & 1<\alpha<2 \\
\Theta\left(n^{-1} \log ^{2} n\right) & \alpha=2 \\
\Theta\left(n^{-1}\right) & \alpha>2
\end{array},\right.
$$

where $\alpha>1$ is the shape parameter of the power-law degree distribution. ${ }^{1}$ This analysis shows that GED is asymptotically uncorrelated for all values of $\alpha>1$ and provides an explanation of the phenomena observed earlier in [51], where GED's clustering decayed to zero while that of the Internet remained constant.

Full derivation of (2) for $1<\alpha<2$ involves a great deal of tedious integration (see Theorem 2), which is unrealistic to perform for each studied method and sometimes even impossible depending on the type of function used to construct each link. To expand the GED result to a wider class of additional methods, we next propose a general framework for reducing degree-based generators to GED in order to obtain their asymptotic assortativity and clustering. We show that as long as the link-existence probability function $\pi$ of a new method can be bounded by that of GED, its correlation asymptotically behaves the same as (1)-(2). We then show that this condition, which we call asymptotic $\pi$-equivalence, holds for PLRG, GLP, BA, and AB. We not only obtain completely novel results on the behavior of degree correlation in PLRG, GLP, and $\mathrm{AB}$, but we also provide an analytical platform of $\pi$-equivalence that can be used to show similar results for any degree-based generator with a power-law degree distribution.

We finish the paper by discussing the implications of results obtained in this work. In particular, our results prove that all existing degree-based generators become uncorrelated as the graph grows in size. This is in stark contrast to networks observed in real life, in which both assortativity and clustering remain constant as the system evolves. This invariance of both the degree distribution and its correlation has an important impact on the design of future topology generators. Specifically, we conjecture that the entire class of degree-based generators is insufficient for capturing the evolving structure of smallworld graphs such as the Internet and illustrate in simulations that link-based generators WIT [51], HOT [18], and SWT [24] are much more effective in keeping node correlation time-invariant. This suggests that the fundamental differences between degree-based and link-based models first observed in this work are indeed significant.

The rest of the paper is organized as follows. Section II explains definitions and related work. Section III derives assortativity and clustering of GED. Section IV introduces the notion of $\pi$-equivalence. Sections V and VI reduce existing topology generators to GED. Section VII briefly compares different graph algorithms to the Internet and Section VIII concludes the paper.

\section{BACKGROUND}

In this section, we overview a small subset of related work, introduce the notation commonly used in this field, and

\footnotetext{
${ }^{1}$ Note that prior results of this nature [5], [8], [9], [15], [25], [47] are available only for the "simple" case of $\alpha \geq 2$, which is not applicable to many existing networks (e.g., the Internet AS graph has $\alpha \approx 1.2$ ).
}

mention several well-known models that we study later in the paper.

Assume an undirected connected graph $G=(\mathcal{V}, \mathcal{E})$ with vertex set $\mathcal{V}$ and edge set $\mathcal{E}$. Suppose that the graph has $|\mathcal{V}|=n$ nodes, whose degrees are given by $d_{1}, \ldots, d_{n}$. For asymptotically large $n \rightarrow \infty$, we treat node degree $d$ as a continuous random variable with $\mathrm{CDF} F(x)=P(d<x)$ and replace all degree-based summations with corresponding integrals. Since we solely focus on scale-free graphs, the CDF function is assumed to be Pareto $F(x)=1-(\beta / x)^{\alpha}$, where $\beta>0$ is the scale parameter and $\alpha>1$ is the shape parameter. Denote by $i \leftrightarrow j$ the event of link $(i, j)$ being present in $\mathcal{E}$ and by $E\left[d^{k}\right]$ the $k$-th moment of node degree in graph $G$.

\section{A. Degree Correlation}

The assortativity coefficient $r(G)$ characterizes two-node degree correlation and measures the extent to which nodes connect preferentially to other nodes with similar degrees [32]. The expectation of $r(G)$ is given by:

$$
E[r(G)]=\frac{E^{2}[d] \sum_{x} \sum_{y} x y \omega(x, y)-E^{2}\left[d^{2}\right]}{E[d] E\left[d^{3}\right]-E^{2}\left[d^{2}\right]},
$$

where $\omega(x, y)$ is defined as the probability that two arbitrary connected nodes have degree $x$ and $y$, respectively [16]:

$$
\omega(x, y)=P\left(d_{i}=x, d_{j}=y \mid i \leftrightarrow j\right) .
$$

Graphs with positive values of $r(G)$ are so-called assortative, graphs with negative $r(G)$ are called disassortative, and graphs with $r(G)=0$ are called uncorrelated.

The most widely-used metric for three-node degree correlation is the clustering coefficient $\gamma(G)$, which quantifies how likely the neighbors of a node are to be connected to each other. Suppose that a given node $i$ is contained in $T_{i}$ triangles. Recall from [52] that the clustering coefficient $\gamma_{i}$ of node $i$ (as long as degree $d_{i}$ is at least two) is defined as the ratio of $T_{i}$ to the maximum number of such triangles:

$$
\gamma_{i}=\frac{T_{i}}{d_{i}\left(d_{i}-1\right) / 2}, d_{i} \geq 2,
$$

and the clustering coefficient $\gamma(G)$ of the graph is then defined to be the average of $\gamma_{i}$ over all nodes $i$ with degree $d_{i} \geq 2$ :

$$
\gamma(G)=\frac{\sum_{i \in \mathcal{V}-\mathcal{V}^{(1)}} \gamma_{i}}{|\mathcal{V}|-\left|\mathcal{V}^{(1)}\right|},
$$

where $\mathcal{V}^{(1)}$ is the set of both degree-zero and degree-one nodes in $G$, i.e., $\mathcal{V}^{(1)}=\left\{j \in \mathcal{V}: d_{j} \leq 1\right\}$. Note that other definitions of the clustering coefficient exist in [9], [46], [54], but they are not studied here.

The combination of assortativity and clustering has been used in characterizing real networks [22], [35]. For power-law networks with shape parameter $\alpha>2$, the following results have been established [8], [15], [33]:

$$
E[r(G)]=0, \quad E[\gamma(G)]=\frac{\left(E\left[d^{2}\right]-E[d]\right)^{2}}{n E^{3}[d]} .
$$

However, little is known about the case of $1<\alpha \leq 2$ commonly found in real networks (e.g., the Internet). The 
closest analysis to tackling this issue is given in [15], which obtains $E[\gamma(G)]$ as a complex summation that allows only numerical computation and provides no qualitative asymptotic behavior in the limit of large $n$. In this paper, we solve this problem and establish the trend of $E[\gamma(G)]$ for all $\alpha>1$.

\section{B. Graph Models}

In this section, we briefly mention several graph models that produce power-law degrees. For a complete literature survey, we refer readers to [16], [33], [38].

1) Non-Evolving Models: In this category, we mention several generators that do not grow (evolve) the network over time. One of the simplest power-law graph construction models is called Given Expected Degree (GED) [14], a specialized algorithm of fitness models [8], [12], [20], [45]. In GED, a sequence of weights $\left\{w_{i}\right\}$ is first generated according to a Pareto distribution and then each edge $(i, j)$ is created with independent probability:

$$
p_{i j}=\min \left(\frac{w_{i} w_{j}}{D}, 1\right),
$$

where $D=\sum_{k=1}^{n} w_{k}$.

A similar graph construction method called Power-Law Random Graph (PLRG) [1], or more generally configuration models, which have been extensively studied in [6], [27], [30], [31], [34], [36], and later extended in [29], [42]. PLRG replicates each node $i$ exactly $w_{i}$ times and then places random edges between the replicated nodes with equal probability.

Additional non-evolving generators include random geometric graphs [24] and rewired small-world (Watts) networks that exhibit heavy-tailed degree distributions [39], [52].

2) Evolving Models: Next, we review several models that grow graph sizes over time. Since Barabási's revival of this direction in 1999, evolution models have taken the center stage of modern topology modeling; however, the earliest evolving model can be traced back to Simon's work in 1955 [10], [43], [44]. Several classes of generators can be described under the umbrella of Generalized Linear Preference (GLP) [11], in which the system grows by adding new links or joining new nodes at each time step. The linking decision is based on the the preferential-attachment function $p_{i}(t)$, which is the probability of node $i$ to be chosen at time $t$ :

$$
p_{i}(t)=\frac{d_{i}(t)-\lambda}{\sum_{k=1}^{n(t)}\left(d_{k}(t)-\lambda\right)},
$$

where shift-parameter $\lambda \in[-\infty, 1]$ and the degree distribution is Pareto with shape $\alpha=2-\lambda \in[1, \infty)$. Similar attachment functions are used in $\mathrm{BA}[4]$ and $\mathrm{AB}$ [2] with certain changes to $\lambda$ and the algorithm for creating new links. Variants of (9) are also used in non-linear preferential-attachment [26], [54] and multi-scaling fitness models [7].

\section{ANALYSIS OF GED}

Recall that GED [14] assigns random weights $w_{i}$ drawn from the Pareto distribution and then creates each link with probability $p_{i j}$ in (8). Next, we explore the asymptotic behavior of GED's degree correlation and answer the question of whether it can be maintained for large $n$. We then demonstrate that PLRG, BA, AB, GLP, and potentially many other graphs can be modeled under the same analytical umbrella.

\section{A. Link Formation}

We first formalize the relationship between weights and edge existence in $G$. Define $\pi(x, y)$ to be the probability of two nodes being connected given their weights $x$ and $y$ :

$$
\pi(x, y)=P\left(i \leftrightarrow j \mid w_{i}=x, w_{j}=y\right) .
$$

For GED, this function is simply:

$$
\pi(x, y)=\min \left(\frac{x y}{D}, 1\right)
$$

where $D=\sum_{k=1}^{n} w_{k}$ is the total degree weight in the graph. ${ }^{2}$

Next, notice that the distribution of $D$ is given by an $n$-fold convolution of Pareto distributions, which makes the analysis of degree correlation intractable. Therefore, most of the derivations below approximate $D$ with its expectation, i.e., $D=n E\left[w_{i}\right]$.

\section{B. Two-Node Correlation - $E[r(G)]$}

It has been shown in [8] that $\omega(x, y)$ in (3) can be described in terms of $\pi(x, y)$ as:

$$
\omega(x, y)=\frac{n f(x) f(y) \pi(x, y)}{E[d]},
$$

where $f(x)$ is the PDF of weights. Substituting (12) into (3) and replacing the summations with integrals, we can express $E[r(G)]$ in terms of $\pi(x, y)$ :

$$
E[r(G)]=\frac{E[d] \rho-E^{2}\left[d^{2}\right]}{E[d] E\left[d^{3}\right]-E^{2}\left[d^{2}\right]},
$$

where $\rho$ is given by:

$$
\rho=n \iint x y \pi(x, y) f(x) f(y) d x d y .
$$

Note that for finite $n$, all node degree is no larger than $n-1$ and higher moments of $d$ in (13) are finite. This leads to the next result that expands the integral in $\rho$ and derives the asymptotic trend of $E\left[d^{2}\right]$ and $E\left[d^{3}\right]$ for Pareto distributions with $\alpha \leq 2$.

Theorem 1: The expected assortativity coefficient of GED graphs is asymptotically:

$$
E[r(G)]=\left\{\begin{array}{ll}
\Theta\left(-n^{1-\alpha}\right) & 1<\alpha<2 \\
\Theta\left(-n^{-1} \log ^{2} n\right) & \alpha=2 \\
0 & \alpha>2
\end{array},\right.
$$

where $\alpha$ is the shape parameter of the power-law weight distribution.

\footnotetext{
${ }^{2}$ The original model described in [14] does not have the min function as in (11), but instead requires that $w_{i} w_{j}<D$. Since this constraint does not hold in the Internet, we replace the inequality with the min function. A recent extension of the original model has been proposed in [8] to construct correlated graphs, however, their method is different from (11) and thus orthogonal to our discussion.
} 


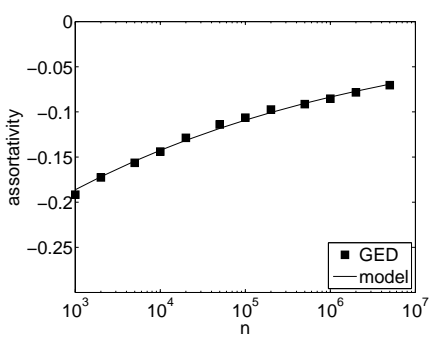

(a) $\alpha=1.2$

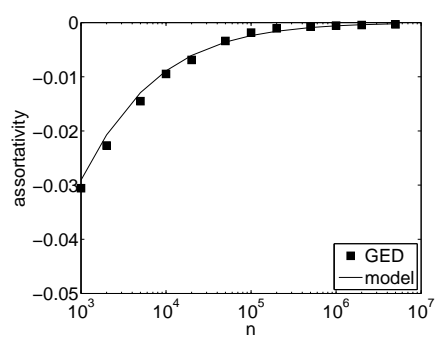

(b) $\alpha=2$
Fig. 1. Model (15) and $E[r(G)]$ in GED simulations. All cases use 10,000 iterations.

Proof: First, we examine the double integral $\rho$. To expand the min function, we split (14) into two parts $\rho_{1}$ and $\rho_{2}$ such that $\rho=\rho_{1}+\rho_{2}$, where:

$$
\rho_{1}=\int_{\beta}^{D} \int_{\beta}^{\frac{D}{x}} \operatorname{nxyf}(x) f(y) \frac{x y}{D} d y d x,
$$

and

$$
\rho_{2}=\int_{\beta}^{D} \int_{\frac{D}{x}}^{D} \operatorname{nxyf}(x) f(y) d y d x .
$$

For $1<\alpha<2$, we obtain $\rho_{1}=\Theta\left(n^{2-\alpha} \log n\right)$ and $\rho_{2}=\Theta\left(n^{2-\alpha} \log n\right)$, from which it follows that $\rho=$ $\Theta\left(n^{2-\alpha} \log n\right)$ for $1<\alpha<2$. For $\alpha=2$, we have $\rho_{1}=\Theta\left(\log ^{2} n\right)$ and $\rho_{2}=\Theta(\log n)$, which establishes that $\rho=\Theta\left(\log ^{2} n\right)$ for $\alpha=2$. For $\alpha>2$, we can omit the min function in the double integral and replace it with $x y / D$ to obtain $\rho=E^{2}\left[d^{2}\right] / E[d]$.

Next, we consider degree moments $E\left[d^{2}\right]$ and $E\left[d^{3}\right]$ in the limit of large $n$ for $1<\alpha \leq 2: E\left[d^{2}\right]=\Theta\left(n^{2-\alpha}\right)$ and $E\left[d^{3}\right]=\Theta\left(n^{3-\alpha}\right)$ for $1<\alpha<2, E\left[d^{2}\right]=\Theta(\log n)$ and $E\left[d^{3}\right]=\Theta(n)$ for $\alpha=2$. Combining with (13), we establish the first two lines in (15).

For $\alpha>2$, substituting $\rho=E^{2}\left[d^{2}\right] / E[d]$ into (13) leads to $E[r(G)]=0$, which is the third line in (15).

To verify the model, we constructed 10,000 GED graphs with shape parameters $\alpha=1.2$ and $\alpha=2$ and extracted the corresponding average assortativity coefficients. In Fig. 1, we plot the curve of $E[r(G)]$ and the corresponding model (15), where we obtain the constant by fitting the model to the actual value for the smallest $n$ used in the simulations. As the figure shows, the model matches simulations well. The result (15) shows that for finite $n$, GED graphs are expected to be disassortative, but as $n \rightarrow \infty$, two-node degree correlation disappears regardless of shape $\alpha$. It is also worth noting that for different $\alpha$, the assortativity coefficient decays to zero at different rates as specified in (15) - the heavier the tail, the slower the decay.

\section{Three-Node Correlation $-E[\gamma(G)]$}

In what follows, we reduce the problem of deriving $E[\gamma(G)]$ to finding the average clustering coefficient of nodes with a given weight. The first lemma indicates that the expected clustering of a single node $i$ (averaged over all possible weight assignments in the graph) is sufficient for establishing $E[\gamma(G)]$.
Lemma 1: In any graph $G$, the expected clustering $E[\gamma(G)]$ is:

$$
E[\gamma(G)]=E\left[\gamma_{i} \mid d_{i} \geq 2\right],
$$

where $i$ is the index of any node in graph $G$.

Proof: Let $N_{2}$ be the number of nodes in the constructed graph $G$ whose degree is at least 2, i.e., $N_{2}=|\mathcal{V}|-\left|\mathcal{V}^{(1)}\right|$. From the definition in (6), the expected clustering $E[\gamma(G)]$ is given by:

$$
E[\gamma(G)]=E\left[\frac{\sum_{i \in \mathcal{V}-\mathcal{V}^{(1)}} \gamma_{i}}{N_{2}}\right]
$$

where $\gamma_{i}$ is the clustering of node $i$. Conditioning on $N_{2}=y$, we can write:

$$
E\left[\gamma(G) \mid N_{2}=y\right]=\frac{\sum_{i=1}^{y} E\left[\gamma_{i} \mid N_{2}=y, d_{i} \geq 2\right]}{y} .
$$

Notice that the summation has exactly $y$ terms and that $E\left[\gamma_{i} \mid N_{2}=y, d_{i} \geq 2\right]$ is the same for all $i$. Therefore, (20) becomes:

$$
E\left[\gamma(G) \mid N_{2}=y\right]=E\left[\gamma_{i} \mid N_{2}=y, d_{i} \geq 2\right],
$$

which establishes (18) by unconditioning $N_{2}$ in (21).

Next, expanding $E\left[\gamma_{i} \mid d_{i} \geq 2\right]$, we yield a more convenient expression for $E[\gamma(G)]$ in the next lemma.

Lemma 2: The expected clustering of a GED graph is given by:

$$
E[\gamma(G)]=\int E[\gamma(x)] f\left(x \mid d_{i} \geq 2\right) d x
$$

where $E[\gamma(x)]=E\left[\gamma_{i} \mid w_{i}=x, d_{i} \geq 2\right]$ is the expected clustering of nodes with weight $x$ and $f\left(x \mid d_{i} \geq 2\right)$ is the conditional PDF of weight $w_{i}$ assigned to node $i$ given that its degree $d_{i} \geq 2$.

Proof: Assume that $j$ and $k \neq j$ are any two neighbors of node $i$ (these neighbors exist since $d_{i} \geq 2$ ). Further, assume that $w_{i}$ is the random weight of node $i$ and $d_{i}$ is its random degree after the graph is built. Then, we can condition on $d_{i}=d$ and expand $E\left[\gamma_{i} \mid d_{i}=d \geq 2\right]$ to become:

$$
\frac{2}{d(d-1)} \sum_{j, k} P\left(T_{i j k}=1 \mid i \leftrightarrow j, i \leftrightarrow k, d_{i}=d \geq 2\right) .
$$

Since the summation in (23) is taken over all possible triangles covering node $i$, it contains exactly $d(d-1) / 2$ terms. Given that weights $w_{j}$ and $w_{k}$ are assigned randomly, each of the triangles has the same probability of existence when averaged over all possible weight assignments, which leads to:

$$
E\left[\gamma_{i} \mid d_{i}=d \geq 2\right]=P\left(j \leftrightarrow k \mid i \leftrightarrow j, i \leftrightarrow k, d_{i}=d \geq 2\right) .
$$

Relaxing the condition on $d_{i}$ gives:

$$
\begin{aligned}
E\left[\gamma_{i} \mid d_{i} \geq 2\right] & =\sum_{d=2}^{n} E\left[\gamma_{i} \mid d_{i}=d \geq 2\right] P\left(d_{i}=d \mid d_{i} \geq 2\right) \\
& =P\left(j \leftrightarrow k \mid i \leftrightarrow j, i \leftrightarrow k, d_{i} \geq 2\right)
\end{aligned}
$$

Thus, $E[\gamma(G)]$ can be reduced to computing the probability that triangle $(i j k)$ exists, conditioned on the fact that $j$ and $k$ are neighbors of $i$ and degree $d_{i} \geq 2$. 


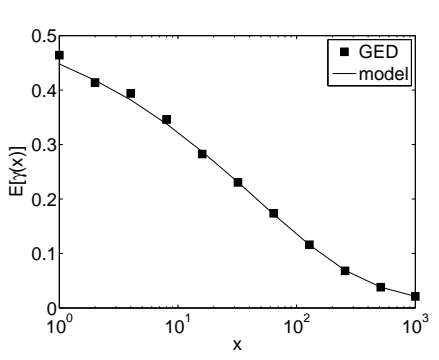

(a) $\alpha=1.2$

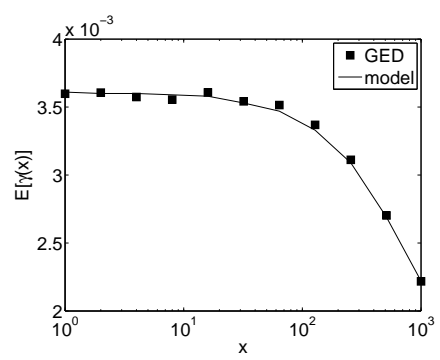

(b) $\alpha=3$
Fig. 2. Verification of (28) in GED simulations with 1,000 nodes and 10,000 iterations).

Finally, noticing that (24) can be obtained from $E[\gamma(x)]$ by relaxing the condition $w_{i}=x$ and integrating over all possible weights $x$ given that $d_{i} \geq 2$, we get:

$$
E\left[\gamma_{i} \mid d_{i} \geq 2\right]=\int E[\gamma(x)] f\left(x \mid d_{i} \geq 2\right) d x,
$$

where $f\left(x \mid d_{i} \geq 2\right)$ is the conditional density of weight $w_{i}$ of node $i$ given that its degree $d_{i} \geq 2$. Since $E[\gamma(G)]=$ $E\left[\gamma_{i} \mid d_{i} \geq 2\right]$, we obtain (22).

To solve (22), we need to derive $f\left(x \mid d_{i} \geq 2\right)$. From Bayes' formula $P(A \mid B)=P(B \mid A) P(A) / P(B)$, we get:

$$
f\left(x \mid d_{i} \geq 2\right)=\frac{P\left(d_{i} \geq 2 \mid w_{i}=x\right) f(x)}{P\left(d_{i} \geq 2\right)},
$$

which can be easily computed knowing that the distribution of $d_{i}$ is Poisson with mean $w_{i}=x$ [8]:

$$
P\left(d_{i}=k \mid w_{i}=x\right)=\frac{x^{k} e^{-x}}{k !} .
$$

The last missing piece toward solving (22) is to develop a formula for $E[\gamma(x)]$.

\section{Weight-Specific Clustering}

Recall that $E[\gamma(x)]=E\left[\gamma_{i} \mid w_{i}=x, d_{i} \geq 2\right]$ is the conditional expectation of $\gamma_{i}$ for a node with weight $w_{i}=x$ and degree at least two. Expanding this metric by additionally conditioning on weights of neighboring nodes $j$ and $k$ gives the following lemma.

Lemma 3: The expected clustering of a node with weight $x$ is given by:

$$
E[\gamma(x)]=\frac{\phi(x)}{\psi^{2}(x)}
$$

where

$$
\begin{aligned}
& \phi(x)=\iint \pi(u, v) \pi(x, u) \pi(x, v) f(u) f(v) d u d v, \\
& \psi(x)=\int \pi(x, u) f(u) d u,
\end{aligned}
$$

and $f(u)$ is the PDF of weights.

Proof: Conditioning on the weights of $j$ and $k$, we have:

$$
\begin{aligned}
E[\gamma(x)] & =\iint \pi(u, v) f\left(u \mid i \leftrightarrow j, w_{i}=x\right) \\
& \times f\left(v \mid i \leftrightarrow k, w_{i}=x\right) d u d v .
\end{aligned}
$$

where $f\left(u \mid i \leftrightarrow j, w_{i}=x\right)$ and $f\left(v \mid i \leftrightarrow k, w_{i}=x\right)$ are respectively the conditional PDFs of the weights randomly assigned to $j$ and $k$. Note that we must use conditional PDFs because weights $w_{j}, w_{k}$ are dependent ${ }^{3}$ on the fact that $w_{i}=x$ and node $i$ is their neighbor.

To derive the integral in (30), we need the conditional density functions of $w_{j}$ and $w_{k}$ given that the weight of $i$ is $x$. Define $\tau(x, u)=f\left(u \mid i \leftrightarrow j, w_{i}=x\right)$. Then, using Bayes' formula, we have:

$$
\begin{aligned}
\tau(x, u) & =\frac{P\left(i \leftrightarrow j, w_{i}=x \mid w_{j}=u\right) f(u)}{P\left(i \leftrightarrow j, w_{i}=x\right)} \\
& =\frac{P\left(i \leftrightarrow j \mid w_{i}=x, w_{j}=u\right) f(x) f(u)}{P\left(i \leftrightarrow j \mid w_{i}=x\right) f(x)} \\
& =\frac{\pi(x, u) f(u)}{\psi(x)} .
\end{aligned}
$$

The last equality (31) comes from the fact that $P\left(i \leftrightarrow j \mid w_{i}=\right.$ $\left.x, w_{j}=u\right)=\pi(x, u)$. It also follows that:

$$
P\left(i \leftrightarrow j \mid w_{i}=x\right)=\int \pi(x, u) f(u) d u=\psi(x) .
$$

Substituting (31) into (30), the result follows immediately.

To verify (28), we created 10,000 GED graphs with $n=$ 1,000 in which one node always had weight $w_{i}=x$ and examined its average clustering coefficient $E\left[\gamma_{i} \mid w_{i}=x, d_{i} \geq\right.$ 2 ]. Simulation results are compared to (28) in Fig. 2, which shows that model (28) matches simulations very accurately.

To obtain $E[\gamma(x)]$ in GED, we first need $\psi(x)$, which can be achieved by expanding the integral in (29).

Lemma 4: For a GED graph with power-law weights, the probability that a link $(i, j)$ exists given that $w_{i}=x$ is asymptotically:

$$
\psi(x)=\frac{x(1-o(1))}{n} .
$$

Proof: Substituting $\pi(x, u)$ into (29), we have:

$$
\psi(x)=\int \min \left(\frac{x u}{D}, 1\right) f(u) d u .
$$

For $x \leq \frac{D}{\beta}$, the integral range in (34) can be divided into two parts, $\left[\beta, \frac{D}{x}\right]$ and $\left[\frac{D}{x}, \infty\right]$, which leads to:

$$
\psi(x)=\int_{\beta}^{\frac{D}{x}} \frac{x u}{D} f(u) d u+\int_{\frac{D}{x}}^{\infty} f(u) d u .
$$

Expanding (35), we have:

$$
\psi(x)=\frac{\alpha \beta x}{D(\alpha-1)}-\frac{\beta^{\alpha} x^{\alpha}}{D^{\alpha}(\alpha-1)} .
$$

Since $D=n E\left[w_{i}\right]=n \alpha \beta /(\alpha-1)$ is sufficiently large, the second term in (36) is negligible compared to the first term. Therefore, we have:

$$
\psi(x)=\frac{x}{n}\left(1-\frac{(\alpha-1)^{\alpha-1} x^{\alpha-1}}{n^{\alpha-1} \alpha^{\alpha}}\right)=\frac{x(1-o(1))}{n},
$$

which completes the proof.

\footnotetext{
${ }^{3}$ For example, if $w_{i}$ is very small and link $(i, j)$ exists, there is a much higher probability that weight $w_{j}$ is large compared to the case where we
} knew nothing about $w_{i}$. 
The most difficult element in deriving $E[\gamma(x)]$ for GED is to formulate the asymptotics of $\phi(x)$, which we present in the next three lemmas for three different cases of $\alpha$.

Lemma 5: For a GED graph with power-law weights and $1<\alpha<2, \phi(x)$ is asymptotically:

$$
\phi(x)= \begin{cases}\Theta\left(x^{2} n^{-\alpha-1} \log n\right) & x \in[\beta, \sqrt{D}) \\ \Theta\left(x n^{-\alpha}\right) & x \in\left[\sqrt{D}, \frac{D}{\beta}\right) . \\ \Theta\left(n^{-1}\right) & x \in\left[\frac{D}{\beta}, \infty\right)\end{cases}
$$

Proof: See Appendix I.

Lemma 6: For a GED graph with power-law weights and $\alpha=2, \phi(x)$ is asymptotically:

$$
\phi(x)= \begin{cases}\Theta\left(x^{2} n^{-3} \log ^{2} n\right) & x \in[\beta, \sqrt{D}) \\ \Theta\left(x^{3} n^{-3}\right) & x \in\left[\sqrt{D}, \frac{D}{\beta}\right) . \\ \Theta\left(n^{-1}\right) & x \in\left[\frac{D}{\beta}, \infty\right)\end{cases}
$$

Proof: See Appendix II.

Lemma 7: For a GED graph with power-law weights and $\alpha>2, \phi(x)$ is asymptotically:

$$
\phi(x)=\left\{\begin{array}{ll}
\Theta\left(x^{2} n^{-3}\right) & x \in\left[\beta, \frac{D}{\beta}\right) \\
\Theta\left(n^{-1}\right) & x \in\left[\frac{D}{\beta}, \infty\right)
\end{array} .\right.
$$

Proof: See Appendix III.

\section{E. Graph Clustering}

Now, we are ready to derive an asymptotic formula for $E[\gamma(G)]$. Combining the results in (26)-(28), we can expand the integral in (22).

Theorem 2: With a power-law distributed weight sequence, the expected GED clustering is asymptotically:

$$
E[\gamma(G)]=\left\{\begin{array}{ll}
\Theta\left(n^{1-\alpha} \log n\right) & 1<\alpha<2 \\
\Theta\left(n^{-1} \log ^{2} n\right) & \alpha=2 \\
\Theta\left(n^{-1}\right) & \alpha>2
\end{array},\right.
$$

where $\alpha$ is the shape parameter of the power-law distribution.

Proof: First recall from (22) and (26) that:

$$
\begin{aligned}
E[\gamma(G)] & =\int_{\beta}^{\infty} E[\gamma(x)] f\left(x \mid d_{i} \geq 2\right) d x \\
& =\int_{\beta}^{\infty} \frac{E[\gamma(x)] P\left(d_{i} \geq 2 \mid w_{i}=x\right) f(x) d x}{P\left(d_{i} \geq 2\right)},
\end{aligned}
$$

where $P\left(d_{i} \geq 2\right)$ can be taken outside the integral since it does not depend on $x$ (in fact, it is a constant). From [8], $d_{i}$ is a Poisson variable with mean $x$, which leads to:

$$
P\left(d_{i} \geq 2 \mid w_{i}=x\right)=1-e^{-x}-x e^{-x} .
$$

The last and most time consuming piece of this theorem is the expansion of $E[\gamma(x)]$, whose asymptotics can be obtained by combining the results of $\phi(x)$ and $\psi(x)$ in the previous lemmas. We split the integration range in (42) into three intervals: $[\beta, \sqrt{D}),\left[\sqrt{D}, \frac{D}{\beta}\right)$, and $\left[\frac{D}{\beta}, \infty\right)$ and calculate each integral separately. Denote by $\delta_{1}, \delta_{2}$, and $\delta_{3}$ the values of these three integrals, respectively.

We start with the case of $1<\alpha<2$. For $x \in[\beta, \sqrt{D})$, we can expand $\delta_{1}$ as:

$$
\delta_{1}=c^{-1} \int_{\beta}^{\sqrt{D}} \xi_{1}(x)\left(1-e^{-x}-x e^{-x}\right) f(x) d x,
$$

where $c=P\left(d_{i} \geq 2\right)$ and $\xi_{1}(x)$ is the leading term in $E[\gamma(x)]$. Combining (33) and (38) with the fact that $E[\gamma(x)]=$ $\phi(x) / \psi^{2}(x)$, it follows that $\xi_{1}(x)=\Theta\left(n^{1-\alpha} \log n\right)$, which is a constant regardless of $x$. Substituting $\xi_{1}(x)$ into (44), we get the following asymptotic form for $\delta_{1}$ :

$$
\begin{aligned}
\delta_{1} & \approx c^{-1} \xi_{1} \int_{\beta}^{\sqrt{D}}\left(1-e^{-x}-x e^{-x}\right) f(x) d x \\
& =\frac{\xi_{1}(c-o(1))}{c}=\Theta\left(n^{1-\alpha} \log n\right) .
\end{aligned}
$$

Similarly, for $x \in\left[\sqrt{D}, \frac{D}{\beta}\right)$, we have (38) that the leading term $\xi_{2}(x)$ in $E[\gamma(x)]$ is $\xi_{2}(x)=\Theta\left(x^{-1} n^{2-\alpha}\right)$, which allows us to derive $\delta_{2}$ as follows:

$$
\begin{aligned}
\delta_{2} & \approx c^{-1} \int_{\sqrt{D}}^{\frac{D}{\beta}} \xi_{2}(x)\left(1-e^{-x}-x e^{-x}\right) f(x) d x \\
& =n^{2-\alpha} c^{-1} \int_{\sqrt{D}}^{\frac{D}{\beta}} x^{-1}\left(1-e^{-x}-x e^{-x}\right) f(x) d x \\
& =O\left(n^{1.5(1-\alpha)}\right) .
\end{aligned}
$$

For $x \in\left[\frac{D}{\beta}, \infty\right)$, we have the leading term $\xi_{3}(x)$ in $E[\gamma(x)]$ is $\xi_{3}(x)=\Theta(1 / n)$ from (38), which leads to:

$$
\delta_{3}=O(1 / n) .
$$

Notice that $E[\gamma(G)]=\delta_{1}+\delta_{2}+\delta_{3}$ and $\delta_{1}$ is asymptotically larger than both $\delta_{2}$ and $\delta_{3}$, which gives the top line of (41).

For $\alpha=2$, the leading term $\xi_{1}(x)$ in $E[\gamma(x)]$ for $x \in$ $[\beta, \sqrt{D})$ is again independent of $x$ from (39), i.e., $\xi_{1}(x)=$ $\Theta\left(n^{-1} \log ^{2} n\right)$, which establishes that:

$$
\delta_{1}=\Theta\left(n^{-1} \log ^{2} n\right) .
$$

Notice from (39) that the leading term $\xi_{2}(x)$ in $E[\gamma(x)]$ for $x \in\left[\frac{D}{\beta}, \infty\right)$ is $\xi_{2}(x)=\Theta\left(x n^{-1}\right)$. We thus obtain a similar result for $\delta_{2}$ from (39):

$$
\delta_{2} \approx c^{-1} \int_{\sqrt{D}}^{\frac{D}{\beta}} x n^{-1}\left(1-e^{-x}-x e^{-x}\right) f(x) d x=O\left(n^{-1.5}\right) .
$$

It is easy to verify that $\delta_{3}=O\left(n^{-1}\right)$ according to (39), from which we directly obtain the second line of (41).

For $\alpha>2$, we have from (40):

$$
\delta_{1}(x)=\delta_{2}(x)=\Theta\left(n^{-1}\right), \quad \delta_{3}(x)=O\left(n^{-1}\right),
$$

which leads to the last line of (41) after discarding insignificant term $\delta_{3}$.

We verified the model by constructing 10,000 GED graphs with shape parameters $\alpha=1.2$ as well as $\alpha=2$ and extracting the corresponding average clustering coefficients, which is plotted in Fig. 3 along with the corresponding model (41). The 


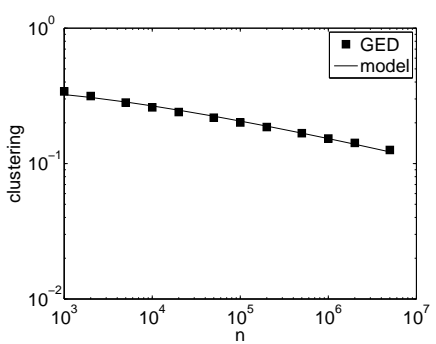

(a) $\alpha=1.2$

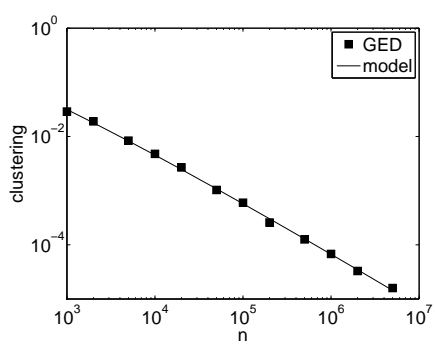

(b) $\alpha=2$
Fig. 3. Model (41) and $E[\gamma(G)]$ in GED simulations. All cases use 10,000 iterations.

figures shows that the model provides an accurate prediction of the asymptotic trend in clustering. Additional simulations show that (41) is accurate for different shape parameters $\alpha$.

The result in (41) not only proves that GED's three-node correlation decays to zero for all values of $\alpha>1$, but also provides the exact asymptotic decay rates.

\section{GENERIC FramewORK OF CORRELATION ANALYSiS}

Notice that the results in (13), (22) do not specify $\pi(x, y)$ and thus can be used to derive the corresponding metric for any degree-based model with a known function $\pi(x, y)$. We next develop a generic framework that allows us to reuse the results derived from (13), (22) in application to PLRG, BA, $\mathrm{AB}$, and GLP.

Consider a graph algorithm $A$ that constructs random graphs $G_{A}$. Denote by $\pi_{A}(x, y)$ the probability of having a link between any pair of nodes with weights $x, y$ in graph $G_{A}$, where weights may be explicitly pre-assigned as in GED or may represent the final degree of each node in the constructed graph. We start with the following definition.

Definition 1: Consider two graph algorithms $A$ and $B$ that operate with the same weight distribution $F(x)$. Define $A$ to be asymptotically $\pi$-equivalent to $B$ if the following holds for all $x \geq 0, y \geq 0$ :

$$
c_{L} \pi_{B}(x, y) \leq \pi_{A}(x, y) \leq c_{U} \pi_{B}(x, y)
$$

where $0<c_{L}<1$ and $c_{U} \geq 1$ are some constants independent of $x, y$, or graph size $n$.

It is easy to verify that $\pi$-equivalence is both symmetric and transitive, which we formulate in the next lemma without proof.

Lemma 8: If $A$ is $\pi$-equivalent to $B$, then $B$ is $\pi$-equivalent to $A$. If $A$ is $\pi$-equivalent to $B$ and $B$ is $\pi$-equivalent to $C$, then $A$ is $\pi$-equivalent to $C$.

The next result follows after straightforward expansion of upper/lower bounds on $\pi(x, y)$ in formulas derived earlier and shows that asymptotic $\pi$-equivalence leads to easy ways of bounding the decay trend of two- and three-node correlation in any graph algorithm.

Theorem 3: If algorithm $A$ is $\pi$-equivalent to $B$, then

$$
\lim _{n \rightarrow \infty} \frac{E\left[r\left(G_{A}\right)\right]}{E\left[r\left(G_{B}\right)\right]}=c_{r}, \quad \lim _{n \rightarrow \infty} \frac{E\left[\gamma\left(G_{A}\right)\right]}{E\left[\gamma\left(G_{B}\right)\right]}=c_{\gamma},
$$

where constants $c_{r}, c_{\gamma}$ are bounded by:

$$
c_{L} \leq c_{r} \leq c_{U}, \quad \frac{c_{L}^{3}}{c_{U}} \leq c_{\gamma} \leq \frac{c_{U}^{3}}{c_{L}}
$$

With the result in Theorem 3, the rest of the paper derives bounds on $\pi(x, y)$ in PLRG, GLP, BA, and AB. Specifically, we show that all of these methods can be reduced to GED through $\pi$-equivalence and thus exhibit the same asymptotic decay rate in $E[r(G)]$ and $E[\gamma(G)]$ in GED.

\section{PLRG}

In what follows, we first give a formula for the linkexistence function $\pi(x, y)$ of PLRG and then study PLRG under the framework developed in the previous section.

Recall that PLRG first pre-assigns a random weight $w_{i}$ drawn from a power-law distribution to each node $i$, generates $w_{i}$ virtual copies of each node $i$, and then uniformly matches these virtual nodes to establish the actual links [1]. To make sure that the resulting degree $d_{i}$ does not exceed the preassigned weights $w_{i}$, virtual nodes paired up during the process are immediately removed from the system.

\section{A. Derivation of $\pi(x, y)$}

To understand the statement of the following lemmas, define $M_{i j}$ and $L_{i j}$ to be random variables that specify the number of edges between nodes $i$ and $j$ with and without counting duplicate links, respectively, at the end of the graph formation process. It follows that:

$$
L_{i j}=\left\{\begin{array}{ll}
1 & M_{i j} \geq 1 \\
0 & M_{i j}=0
\end{array} .\right.
$$

Let the node matching process in PLRG start at time 1 and finish at time $D$ (recall that $D$ is twice the number of edges), where the node chosen at time $2 k-1$ is paired up with the node chosen at time $2 k$. Denote by $p_{i j}(t)$ the probability of forming a new edge between nodes $i$ and $j$ at time $t$. We first show in the next lemma that $p_{i j}(t)$ in PLRG does not depend on time $t$ and then apply this result to deriving a more interesting expression for $E\left[L_{i j}\right]=P(i \leftrightarrow j)=\pi\left(w_{i}, w_{j}\right)$.

Lemma 9: The probability $p_{i j}(t)$ of forming a new link between $i$ and $j$ is independent of $t$ :

$$
p_{i j}(t)=\frac{2 w_{i} w_{j}}{D^{2}}
$$

where $D=\sum_{k=1}^{n} w_{k}$.

Proof: First, we examine how the number of virtual copies $w_{i}(t)$ of node $i$ decreases as a function of $t$. According to the description of PLRG [1], $w_{i}(0)=w_{i}$ and each copy at time $t$ is chosen with probability $p_{i}(t)=w_{i}(t) /(D-t)$. This leads to a stochastic difference equation on $w_{i}(t)$ :

$$
w_{i}(t)-w_{i}(t-1)=\left\{\begin{array}{ll}
-1 & \text { w.p. } p_{i}(t) \\
0 & \text { w.p. } 1-p_{i}(t)
\end{array} .\right.
$$

Applying continuum theory [3] to (56), we get:

$$
\frac{\partial w_{i}(t)}{\partial t}=-\frac{w_{i}(t)}{D-t}
$$




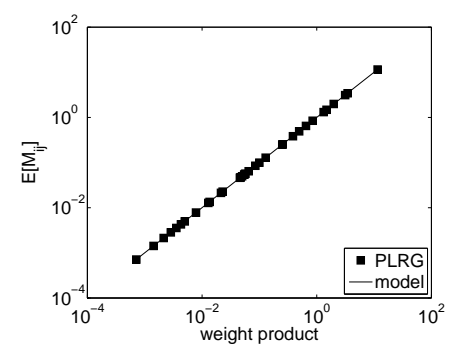

(a) $E\left[M_{i j}\right]$

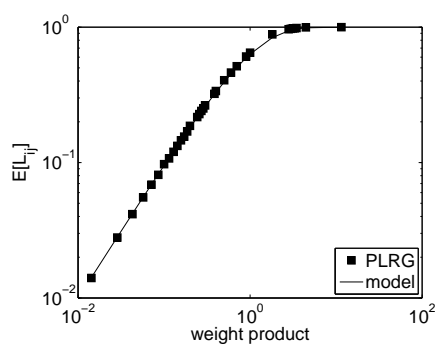

(b) $E\left[L_{i j}\right]$

Fig. 4. Simulations of PLRG $(\alpha=1.2)$. All cases use 30,000 iterations.

Combining with the initial condition $w_{i}(0)=w_{i}$, where $w_{i}$ is the initially assigned weight, it follows that:

$$
w_{i}(t)=(D-t) \frac{w_{i}}{D}
$$

Noticing that matching operations only happen at instants $t=2 k$ (for $k=1,2 \ldots$ ), we have $p_{i j}(2 k-1)=0$. At $t=2 k$, a new link is created between $i, j$ if both nodes $i$ and $j$ are chosen during the two most-recent steps. It follows that for $t=2 k$ :

$$
p_{i j}(t)=\frac{w_{i}(t-1) w_{j}(t)}{(D-t+1)(D-t)}+\frac{w_{j}(t-1) w_{i}(t)}{(D-t+1)(D-t)},
$$

which combined with (58) leads to (55).

The result in (55) specifies the rate of accumulating links between $i$ and $j$ and leads to the following result stating the expectations of $M_{i j}$ and $L_{i j}$.

Theorem 4: The expected number of links between $i$ and $j$ is given by:

$$
E\left[M_{i j}\right]=\frac{w_{i} w_{j}}{D}, \quad E\left[L_{i j}\right]=1-e^{-w_{i} w_{j} / D} .
$$

Proof: It is straightforward to prove the result of $E\left[M_{i j}\right]$ as follows:

$$
E\left[M_{i j}\right]=\sum_{t=0}^{D / 2-1} p_{i j}(t)=\frac{w_{i} w_{j}}{D} .
$$

Next, we focus on $E\left[L_{i j}\right]$. Similar to our earlier notation, let $L_{i j}(t)$ indicate the existence of link $(i, j)$ at time $t$ (i.e., $L_{i j}(t)=1$ if the link exists and 0 otherwise). Notice that $L_{i j}(t)$ is by definition a Bernoulli random variable. Denote by $l_{i j}(t)=E\left[L_{i j}(t)\right]=P\left(L_{i j}=1\right)$ the expectation of $L_{i j}(t)$. Then, notice that link $(i, j)$ exists at time $t$ (i.e., $L_{i j}(t)=1$ ) either because it already existed at time $t-2$ or because it was added during step $t$. It follows that:

$$
l_{i j}(t)=l_{i j}(t-2)+\left(1-l_{i j}(t-2)\right) p_{i j}(t),
$$

where $p_{i j}(t)$ is given in (55). Solving the difference equation and setting $t=D$, we get:

$$
E\left[L_{i j}\right]=l_{i j}(D)=1-\left(1-\frac{2 w_{i} w_{j}}{D^{2}}\right)^{D / 2} .
$$

Since $D$ is large, (60) follows immediately by approximating $(1-z)^{u}$ with $e^{-z u}$ and omitting $o(1)$ terms from the exponent.

To verify the correctness of (60), we constructed 30,000 graphs PLRG and computed the average $M_{i j}$ and $L_{i j}$ of all

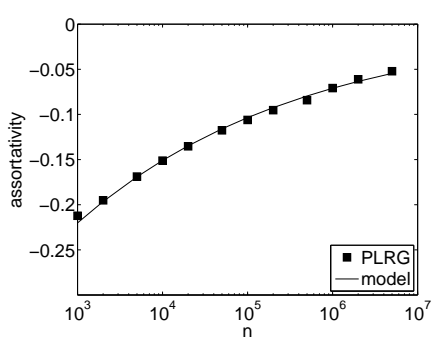

(a) assortativity

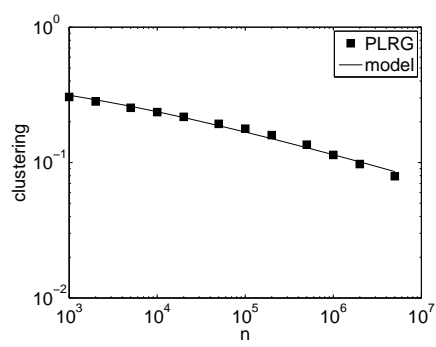

(b) clustering
Fig. 5. Simulations of PLRG ( $\alpha=1.2)$. All cases use 10,000 iterations.

pairs of nodes that have the same weight products. Fig. 4 plots $E\left[M_{i j}\right]$ and $E\left[L_{i j}\right]$ against the normalized weight product $w_{i} w_{j} / D$, indicating that model (60) follows the actual values accurately.

Note that the distribution of $L_{i j}$ specifies the link-existence probability function $\pi(x, y)$, which is formulated in the next corollary.

Corollary 1: For PLRG, the link-existence probability function $\pi(x, y)$ is given by:

$$
\pi(x, y)=1-e^{-x y / D} .
$$

Note that for $x y / D \approx 0$, PLRG is identical to GED. However, as $x y$ increases, the two algorithms exhibit diverging link formation probabilities and thus produce graphs with numerically different assortativity and clustering.

\section{B. Bounds on $\pi(x, y)$}

With the result in (64), we next show that PLRG's correlation decays to zero at the same rate as that in GED.

Theorem 5: PLRG is $\pi$-equivalent to GED.

Proof: It is easy to verify that for all $x>0, y>0$ :

$$
\pi_{P L R G}(x, y) \leq \min \left(\frac{x y}{D}, 1\right) .
$$

In addition, for all $x>0, y>0$ :

$$
\pi_{P L R G}(x, y) \geq(1-1 / e) \min \left(\frac{x y}{D}, 1\right) .
$$

Therefore, there exist $c_{U}=1$ and $c_{L}=1-1 / e$ that guarantee $\pi_{P L R G}(x, y)$ to be upper- and lower bounded by $c_{U} \pi_{G E D}(x, y)$ and $c_{L} \pi_{G E D}(x, y)$, respectively.

Corollary 2: As graph size $n \rightarrow \infty$, two- and three-node correlation in PLRG decays to zero according to (15) and (41).

Fig. 5 plots the average degree correlation coefficients obtained from simulations of 10,000 PLRG graphs with $\alpha=1.2$ and indicates that the asymptotic models (15) and (41) match the actual very well for PLRG. Numerous additional simulations (omitted for brevity) have been conducted to verify (15) and (41) for other values of $\alpha$ in PLRG.

\section{GLP}

In the category of evolving models, we start with the most general case of GLP and later reduce the obtained result to BA and AB. Recall from [11] that at each time step $t$, with probability $p$ GLP adds $m$ new links or with probability $1-p$ joins a new node with $m$ links. The way of selecting 
existing nodes for building new links or attaching new nodes is specified by the shifted preferential function $p_{i}(t)$ :

$$
p_{i}(t)=\frac{d_{i}(t)-\lambda}{D_{\lambda}(t)},
$$

where $d_{i}(t)$ is the degree of node $i$ at time $t, \lambda \leq 1$ is the shift parameter fed into the algorithm, and $D_{\lambda}(t)=\sum_{k=1}^{n(t)}\left(d_{k}(t)-\right.$ $\lambda)$ is the total shifted degree.

\section{A. Derivation of $\pi(x, y)$}

In what follows, we assume that all nodes join the system sequentially and that each node $i$ arrives at time $t_{i}$. It has been shown in [11] that degree $d_{i}(t)$ of node $i$ follows:

$$
d_{i}(t)=\left(\frac{\alpha t-b}{\alpha t_{i}-b}\right)^{1 / \alpha}(m-\lambda)+\lambda,
$$

where $\alpha$ is the shape of the Pareto degree distribution and $b$ is a constant:

$$
\alpha=\frac{2 m-\lambda(1-p)}{m(1+p)}, \quad b=\frac{m_{0} \lambda}{m(1+p)} .
$$

The total shifted degree $D_{\lambda}(t)$ at time $t$ in GLP graphs is then given by:

$$
D_{\lambda}(t)=2 m t-\left(m_{0}+(1-p) t\right) \lambda=m(1+p)(\alpha t-b) .
$$

Now, consider two nodes $i, j$ in the graph and suppose that $j$ joins after $i$, i.e., $t_{i}<t_{j}$. Denote by $M_{i j}(t)$ the number of duplicate links between $i$ and $j$ accumulated up to time $t$ and by $L_{i j}(t)$ the indicator variable that represents if any link between $i, j$ exists by time $t$ :

$$
L_{i j}(t)=\left\{\begin{array}{ll}
1 & M_{i j}(t) \geq 1 \\
0 & M_{i j}(t)=0
\end{array} .\right.
$$

Next, we apply similar technique as in PLRG to obtain formulas for $M_{i j}(t)$ and $L_{i j}(t)$. Before doing so, we define two auxiliary variables as follows. Denote by $p_{i j}(t)$ the probability for nodes $i, j$ to form a link at time $t$. It follows from (67) that the probability $p_{i j}\left(t_{j}\right)$ upon arrival of node $j$ is given by:

$$
p_{i j}\left(t_{j}\right)=\frac{d_{i}\left(t_{j}\right)-\lambda}{D_{\lambda}\left(t_{j}\right)},
$$

while after $t_{j}$, the probability $p_{i j}(t)$ is determined by:

$$
p_{i j}(t)=2 p \frac{d_{i}(t)-\lambda}{D_{\lambda}(t)} \cdot \frac{d_{j}(t)-\lambda}{D_{\lambda}(t)} .
$$

Further denote by $A_{i j}(t)$ the number of links, counting duplicates, created between $i, j$ at time $t$. It is straightforward to show that $A_{i j}(t)$ is binomial $B\left(m, p_{i j}(t)\right)$ and:

$$
M_{i j}(t)=\sum_{s=t_{j}}^{t} A_{i j}(s) \text {. }
$$

Now, we can prove the next result for $E\left[M_{i j}(t)\right]$.
Theorem 6: Assume that $t_{i}<t_{j}$. For $\alpha \neq 2$, the expected number of duplicated links between $i$ and $j$ in GLP is given by:

$E\left[M_{i j}(t)\right]=\frac{\left(d_{i}(t)-\lambda\right)\left(d_{j}(t)-\lambda\right)}{D_{\lambda}(t)}\left(c_{1}+c_{2}\left(d_{j}(t)-\lambda\right)^{\alpha-2}\right)$,

where $c_{1}$ and $c_{2}$ are constants:

$$
c_{1}=\frac{2 p}{(2-\alpha)(1+p)}, \quad c_{2}=\frac{m \lambda}{(2-\alpha)(m-\lambda)^{\alpha-1}} .
$$

For $\alpha=2$, the expected value of $M_{i j}(t)$ is:

$E\left[M_{i j}(t)\right]=\frac{\left(d_{i}(t)-\lambda\right)\left(d_{j}(t)-\lambda\right)}{D_{\lambda}(t)}\left(c_{3}+c_{4} \log \left(d_{j}(t)-\lambda\right)\right)$,

where $c_{3}$ and $c_{4}$ are constants:

$$
c_{3}=\frac{m}{m-\lambda}+\frac{4 p \log (m-\lambda)}{1+p}, \quad c_{4}=\frac{4 p}{1+p} .
$$

Proof: For simplicity, define the following variables:

$$
\nu_{i}=\left(\alpha t_{i}-b\right)^{1 / a}, \quad \nu_{j}=\left(\alpha t_{j}-b\right)^{1 / \alpha} .
$$

It then follows from (73) that $p_{i j}(t)$ is given by:

$$
p_{i j}(t)=\frac{2 p(m-\lambda)^{2}}{m^{2}(1+p)^{2}} \frac{(\alpha t-b)^{2 / \alpha-2}}{\nu_{i} \nu_{j}} .
$$

Denote by $z_{i j}(t)=E\left[M_{i j}(t)\right]$ the expectation of $M_{i j}(t)$. Taking expectation on both sides of (74), we obtain the following difference equation:

$$
z_{i j}(t)-z_{i j}(t-1)=E\left[A_{i j}(t)\right]=m p_{i j}(t),
$$

with the initial condition from (72):

$$
z_{i j}\left(t_{j}\right)=\frac{m(m-\lambda) \nu_{j}}{m \nu_{i} \nu_{j}^{\alpha}(1+p)} .
$$

Applying continuum theory to (81) and solving the differential equation

$$
\frac{\partial z_{i j}(t)}{\partial t}=m p_{i j}(t)=\frac{2 m p(m-\lambda)^{2}}{m^{2}(1+p)^{2}} \frac{(\alpha t-b)^{2 / \alpha-2}}{\nu_{i} \nu_{j}},
$$

we establish that:

$$
z_{i j}(t)=c(t)+c_{0},
$$

where $c(t)$ and $c_{0}$ are determined for two cases of $\alpha$ as follows.

For $\alpha \neq 2$, we solve (83) to establish that:

$$
c(t)=\frac{2 m p(m-\lambda)^{2}}{m^{2}(1+p)^{2} \nu_{i} \nu_{j}} \frac{(\alpha t-b)^{2 / \alpha-1}}{2-\alpha} .
$$

Note that from initial condition $c_{0}=z_{i j}\left(t_{j}\right)-c\left(t_{j}\right)$, it follows that:

$$
\begin{aligned}
c_{0} & =\frac{m(m-\lambda) \nu_{j}}{m \nu_{i} \nu_{j}^{\alpha}(1+p)}-\frac{2 m p(m-\lambda)^{2} \nu_{j}^{2-\alpha}}{m^{2}(1+p)^{2}(2-\alpha) \nu_{i} \nu_{j}} \\
& =\frac{m \lambda(1+p)}{m^{2}(1+p)^{2}(2-\alpha) \nu_{i} \nu_{j}^{\alpha-1}} .
\end{aligned}
$$


For $\alpha=2, c(t)$ and $c_{0}$ become:

$$
c(t)=\frac{2 m p(m-\lambda)^{2} \log (\alpha t-b)}{m^{2}(1+p)^{2} \nu_{i} \nu_{j}},
$$

and

$$
\begin{aligned}
c_{0} & =\frac{m(m-\lambda) \nu_{j}}{m \nu_{i} \nu_{j}^{2}(1+p)}-\frac{2 m p(m-\lambda)^{2} \log \left(\alpha t_{j}-b\right)}{m^{2}(1+p)^{2} \nu_{i} \nu_{j}} \\
& =\frac{m(1+p)(m-\lambda)-2 p(m-\lambda)^{2} \log \left(\alpha t_{j}-b\right)}{m^{2}(1+p)^{2}(2-\alpha) \nu_{i} \nu_{j}} .
\end{aligned}
$$

The last step is to replace $\nu_{i}, \nu_{j}$ with $d_{i}(t), d_{j}(t), D_{\lambda}(t)$ in (85)-(86). Combining with (68)-(70), we rewrite (79) to express $\nu_{i}, \nu_{j}$ using $d_{i}(t), d_{j}(t), D_{\lambda}(t)$ :

$$
\begin{aligned}
\nu_{i} & =\frac{m-\lambda}{d_{i}(t)-\lambda}\left(\frac{D_{\lambda}(t)}{m(1+p)}\right)^{1 / \alpha}, \\
\nu_{i} & =\frac{m-\lambda}{d_{j}(t)-\lambda}\left(\frac{D_{\lambda}(t)}{m(1+p)}\right)^{1 / \alpha} .
\end{aligned}
$$

We also express terms $\alpha t-b$ and $\alpha t_{j}-b$ with $d_{i}(t), d_{j}(t)$, and $D_{\lambda}(t)$ using (68)-(70) as follows:

$$
\alpha t-b=\frac{D_{\lambda}(t)}{m(1+p)}, \alpha t_{j}-b=\left(\frac{m-\lambda}{d_{j}(t)-\lambda}\right)^{\alpha} \frac{D_{\lambda}(t)}{m(1+p)} .
$$

Combining (85)-(90) gives the desired result.

Now, it remains to derive $L_{i j}(t)$, which we perform in the next theorem.

Theorem 7: In a GLP graph, $L_{i j}(t)$ is asymptotically Bernoulli with expectation:

$$
E\left[L_{i j}(t)\right]=1-e^{-E\left[M_{i j}(t)\right]},
$$

Proof: Denoting by $l_{i j}(t)=E\left[L_{i j}(t)\right]$ the expectation of $L_{i j}(t)$. Following the same reasoning as in Theorem 4, we have:

$$
l_{i j}(t)=l_{i j}(t-1)+\left(1-l_{i j}(t-1)\right) P\left(A_{i j}(t) \geq 1\right) .
$$

Noticing that $A_{i j}(t)$ is $\operatorname{Binomial}\left(m, p_{i j}(t)\right)$, we have:

$$
P\left(A_{i j}(t) \geq 1\right)=1-\left(1-p_{i j}(t)\right)^{m}=m p_{i j}(t)(1+o(1)),
$$

where the second equality comes from the fact that $p_{i j}(t)$ is sufficiently small as $n \rightarrow \infty$. Note that we omit all $o(1)$ terms in the remainder of this proof. It follows from (83) that:

$$
\frac{\partial l_{i j}(t)}{\partial t}=\left(1-l_{i j}(t)\right) \frac{\partial z_{i j}(t)}{\partial t},
$$

with the initial condition

$$
l_{i j}\left(t_{j}\right)=1-e^{-m p_{i j}\left(t_{j}\right)}=1-e^{-z_{i j}\left(t_{j}\right)},
$$

from which we solve for $l_{i j}(t)$ to establish (91).

To verify models (77) and (91), we generated a GLP graph with $10^{6}$ nodes using parameters $m_{0}=2, m=$ $1.13, p=0.4695, \lambda=0.6$. We fixed $i=1$ and uniformly randomly select node $j$ from the rest nodes and computed the average number of duplicate/non-duplicate links. Fig. 6 plots the obtained $E\left[M_{i j}(t)\right]$ and $E\left[L_{i j}(t)\right]$ against $d_{j}$ from the simulations along with the model values. The figure shows

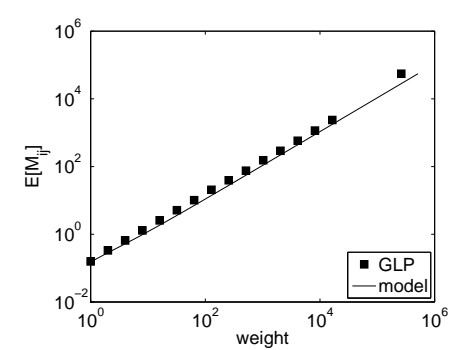

(a) $E\left[M_{i j}\right]$

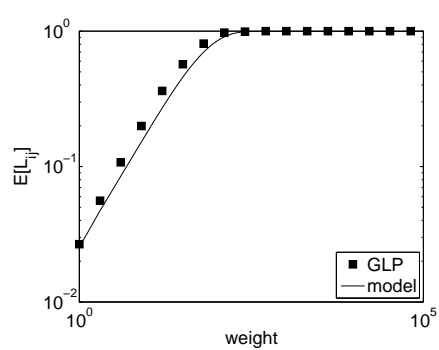

(b) $E\left[L_{i j}\right]$
Fig. 6. Simulations of GLP $(\alpha=1.2)$. All cases use 10, 000 iterations.

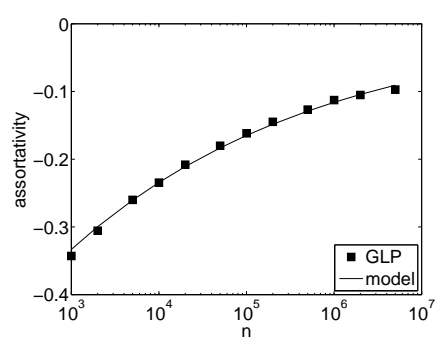

(a) assortativity

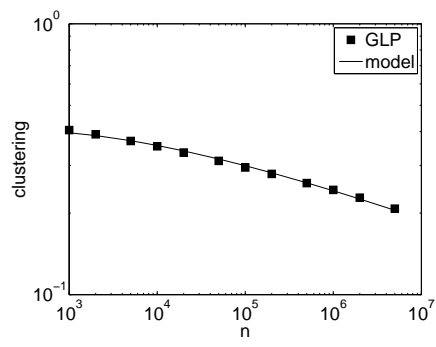

(b) clustering
Fig. 7. Simulations of GLP $(\alpha=1.2)$. All cases use 10,000 iterations.

that models (77) and (91) follow the simulation results pretty accurately.

Notice that the result in (91) assumes that node $j$ arrives after $i$. However, for any pair of randomly chosen nodes $i, j$, their join times satisfy $P\left(t_{i}<t_{j}\right)=P\left(t_{i}>t_{j}\right)=1 / 2$. Further note that we set the weight of each node $i$ to be its shifted degree, i.e., $w_{i}=d_{i}-\lambda$. This leads to the weight distribution $F(x)$ being the same Pareto distribution $1-(x / \beta)^{-\alpha}$ as in GED and allows application of $\pi$-equivalence later.

Corollary 3: In GLP with $\alpha \neq 2$, link-existence probability $\pi(x, y)$ is given by:

$$
\begin{aligned}
\pi(x, y) & =1-1 / 2 \exp \left(-\frac{x y}{D}\left(c_{1}+c_{2} x^{\alpha-2}\right)\right) \\
& -1 / 2 \exp \left(-\frac{x y}{D}\left(c_{1}+c_{2} y^{\alpha-2}\right)\right),
\end{aligned}
$$

and for $\alpha=2$ :

$$
\begin{aligned}
\pi(x, y) & =1-1 / 2 \exp \left(-\frac{x y}{D}\left(c_{3}+c_{4} \log x\right)\right) \\
& -1 / 2 \exp \left(-\frac{x y}{D}\left(c_{3}+c_{4} \log y\right)\right),
\end{aligned}
$$

where $x, y$ are node weights, $D$ is the total weight, $c_{1}, c_{2}$ are given in (76), and $c_{3}, c_{4}$ are in (78).

\section{B. Bounds on $\pi(x, y)$}

Now we use simple exponential functions to bound $\pi(x, y)$. In the next lemma, we only show the results for the case of $\lambda>0$ (i.e., $1<\alpha<2$ ). However, the following results also hold for $\lambda<0$ after reversing the direction of each inequality.

Lemma 10: In GLP with $\lambda>0$, the link-existence probability function $\pi(x, y)$ is bounded as follows:

$$
1-\exp \left(-\frac{x y}{c_{6} D}\right) \leq \pi(x, y) \leq 1-\exp \left(-\frac{x y}{c_{5} D}\right),
$$




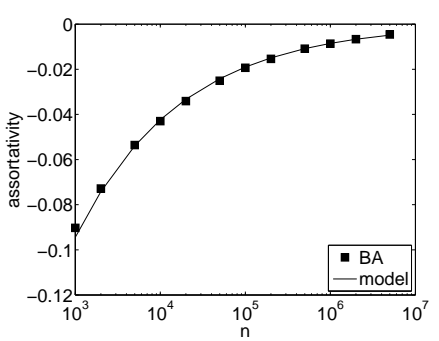

(a) assortativity

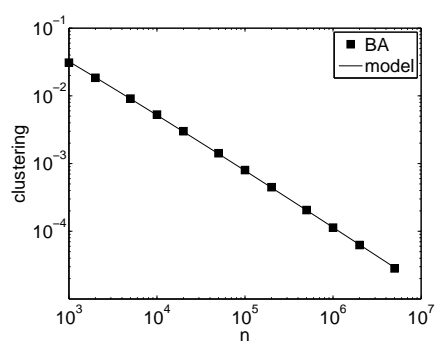

(b) clustering
Fig. 8. Simulations of BA. All cases use 10,000 iterations.

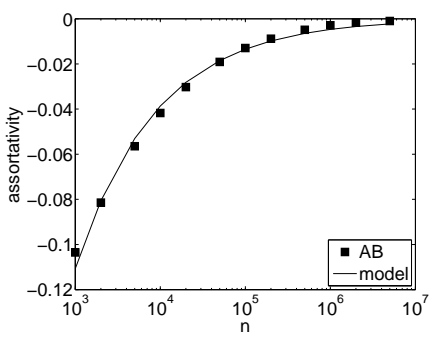

(a) assortativity

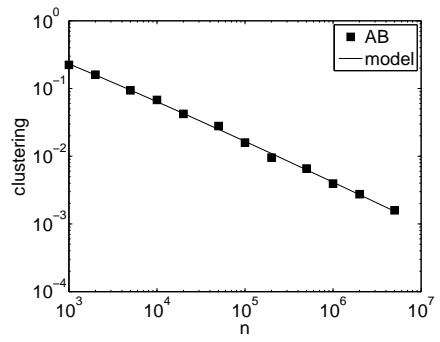

(b) clustering
Fig. 9. Simulations of $\mathrm{AB}(\alpha=1.2)$. All cases use 10,000 iterations.

where $c_{5}, c_{6}$ are constants:

$$
c_{5}=\frac{1}{c_{1}\left(p+\frac{m \lambda(1+p)}{2(m-\lambda)}\right)}, \quad c_{6}=\frac{1}{c_{1} p} .
$$

Proof: Noticing in (96):

$$
c_{2} x^{\alpha-2}=\frac{m \lambda(1+p)}{2(m-\lambda)}\left(\frac{x}{m-\lambda}\right)^{\alpha-2} \leq \frac{m \lambda(1+p)}{2(m-\lambda)},
$$

the upper bound on $\pi(x, y)$ is thus given by:

$$
\pi(x, y) \leq 1-\exp \left(-c_{1}\left(p+\frac{m \lambda(1+p)}{2(m-\lambda)}\right) \frac{x y}{D}\right) .
$$

Further recall that $D_{\lambda}=\left(1-\lambda / E\left[d_{i}\right]\right) D$, which leads to the upper bound in (98).

Considering that $\lambda>0$, we have $1<\alpha<2$ and thus $c_{1}>0, c_{2}>0$. It follows that a natural lower bound on $\pi(x, y)$ is given by:

$$
\pi(x, y) \geq 1-\exp \left(-c_{1} p \frac{x y}{D}\right)
$$

which establishes the lower bound in (98).

The following results show that we can infer the asymptotics of $E[r(G)]$ and $E[\gamma(G)]$ of GLP from those of PLRG and, through transitivity, those of GED.

Theorem 8: GLP is $\pi$-equivalent to PLRG.

Proof: Notice that $\pi_{G L P}(x, y)$ is bounded by the exponential functions shown in (98). It is easy to verify that $c_{5} \leq 1$ and $c_{6} \leq 1$ for $\lambda>0$. It follows that:

$$
1-\exp \left(-\frac{x y}{c_{6} D}\right) \geq 1-\exp \left(-\frac{x y}{D}\right)
$$

and

$$
1-\exp \left(-\frac{x y}{c_{5} D}\right) \leq 1 / c_{5}\left(1-\exp \left(-\frac{x y}{D}\right)\right) \text {. }
$$

Therefore, setting $c_{L}=1, c_{U}=1 / c_{5}$, we get the desired result.

Corollary 4: As graph size $n \rightarrow \infty$, two- and three-node correlation in GLP decays to zero according to (15) and (41).

Again, we verify Corollary 4 by constructing 10,000 GLP graphs and extracting $r(G)$ and $\gamma(G)$. We plot in Fig. 7 the values from simulations as well as from models (15) and (41). The figure displays that the model curves follow the actual values very accurately.

It is interesting to note that while GLP has significantly higher clustering than GED/PLRG according to [11], [51], the results in Corollary 4 for the first time analytically show that GLP is asymptotically the same as GED/PLRG in the limit of large $n$.

\section{Application to $B A$ and $A B$}

Note that BA is a special case of GLP with $p=0$ and $\lambda=0$, from which it follows that $\alpha=2$ and $b=0$. Notice that (96) does not admit $\alpha=2$ since $c_{1}=\infty$. We repeat the derivation of (77)-(91) with $\alpha=2, p=0$, and $\lambda=0$ to yield the following results.

Theorem 9: The link existence probability $\pi(x, y)$ in $\mathrm{BA}$ is identical to that of PLRG given in (64).

Proof: Notice that the rate of increase in $z_{i j}(t)$ is just zero since in BA $p_{i j}(t)=0$ for $t>t_{j}$. Therefore, solving the differential equation (83) with the initial condition (82) establishes that:

$$
z_{i j}(t)=z_{i j}\left(t_{j}\right)=\frac{m}{\nu_{i} \nu_{j}}=\frac{d_{i}(t) d_{j}(t)}{D(t)} .
$$

Applying the same reasoning as in the proof of Theorem 7, we obtain the same $\pi(u, v)$ for BA as in PLRG

Corollary 5: BA is $\pi$-equivalent to PLRG and the following asymptotic decay rates hold for BA: $E[r(G)]=\Theta\left(n^{-1} \log ^{2} n\right)$ and $E[\gamma(G)]=\Theta\left(n^{-1} \log ^{2} n\right)$.

Considering that $\mathrm{AB}$ without edge rewiring is just another special case of GLP, we get the following results by setting $\lambda=-1$ in (98).

Corollary 6: AB (without rewiring) is $\pi$-equivalent to PLRG and its correlation satisfies (15), (41) as $n \rightarrow \infty$.

Not surprisingly, both BA and AB have decaying assortativity and clustering, just as GLP. Simulation results of BA and $\mathrm{AB}$ are given in Fig. 8-9, which show that the asymptotic degree correlation in $\mathrm{BA}$ and $\mathrm{AB}$ graphs can be accurately predicted using (15), (41).

\section{DISCUSSION}

In this section, we compare the evolution of degree correlation given in (15), (41) to that of the Internet AS-level structure. We specifically focus on the subgraphs of the Internet induced by provider-customer links, which have been shown in [21] to exhibit a power-law degree distribution. According to [21], the combination of BGP routing tables from [40], [41] provides a reasonably complete view of the provider-customer links in the Internet.

We first obtain more than 300 snapshots of the Internet topology from BGP routing tables [40], [41], which cover the 


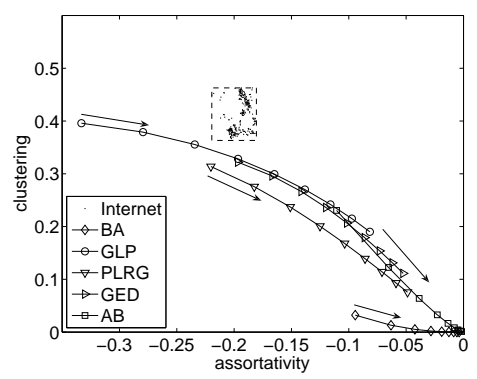

(a) degree-based

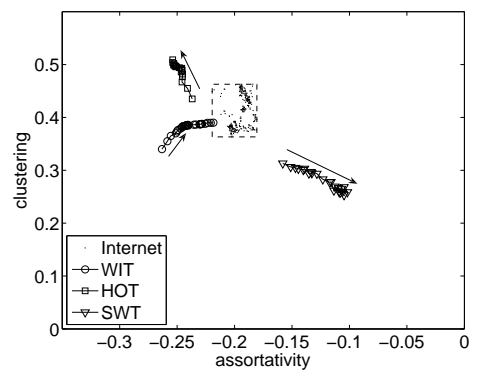

(b) link-based

Fig. 10. Comparison of degree-based and link-based models to the Internet. All markers are obtained from asymptotic models (15), (41) in (a) and from simulations in (b). Arrows indicate the trends in corresponding curves.

last 7 years as the size of the Internet has increased from 4,000 to 23,000 nodes. We then extract degree correlation from these snapshots and plot them in Fig. 10 with $r(G)$ on the $x$-axis and $\gamma(G)$ on the $y$-axis. The figure indicates that both degree correlation coefficients of the Internet, shown as dots inside a small dashed rectangle, do not significantly change over the years, which is consistent with observations in [51].

We also plot in Fig. 10(a) the corresponding metrics computed from (15) and (41) for the studied degree-based algorithms with graph size ranging from $10^{3}$ to $10^{7}$. The figure shows that for small graph sizes, degree correlation of GLP/GED/PLRG is close to that of the Internet; however, all studied degree-based models converge both assortativity and clustering to zero as system size increases. This contradicts the behavior of these metrics in real networks and suggests that attachment decisions of users in small-world graphs may be based on additional factors besides the degree of potential neighbors.

To support this conjecture, we next study three linkbased graph algorithms: Small-World Topologies (SWT) [24], Heuristically Optimized Trade-offs (HOT) [18], and Wealthbased Internet Topology (WIT) [51]. Recall that SWT forms $p$ percent of links using geographic preference and creates the rest of the links using random pairing as in PLRG; HOT models each attachment decision as an optimization problem with the goal of minimizing both the geographical length of potential links and the average number of hops to other nodes in the graph; and WIT adjusts the number of links based on an underlying stochastic wealth process and selects neighbors based on random walks.

We configure SWT/HOT/WIT in simulations to produce power-law degree distributions that are the similar to those observed in the Internet. In SWT, we set $p=0.31$ as in [24]; in HOT, we add two new links to each new node as in [51]; for WIT, we used the parameters of [51]. For each of these three algorithms, we construct 10,000 graphs with $n$ increasing from $10^{3}$ to $10^{7}$ and extract the averaged degree correlation.

Observe from Fig. 10(b) that SWT exhibits decreasing $r(G)$ and $\gamma(G)$ and displays a similar trend as degree-based algorithms, which can be explained by the fact that SWT contains PLRG-like random pairing. In contrast, notice that HOT increases its clustering coefficient as the graph becomes more disassortative, an opposite trend to that of degreebased methods. Both approaches, however, exhibit much less sensitivity to increasing $n$ than degree-based generators and reach a saturation point as $n \rightarrow \infty$, which closely replicates the qualitative behavior of the Internet. Finally, $r(G)$ and $\gamma(G)$ of WIT graphs stabilize around those of the Internet, even reaching and staying inside the dashed rectangle for $n \geq 50,000$.

These results show the significant difference between degree-based and link-based methods, as well as underline the importance of modeling higher-order degree correlation in constructed graphs.

\section{CONCLUSION}

In this paper, we examined several degree-based topology generators and derived the asymptotic shape of their assortativity and clustering as $n \rightarrow \infty$. We found that all studied degreebased generators were virtually uncorrelated for sufficiently large graph size. Our simulations also showed that link-based algorithms [18], [24], [51], which grew the system based on the structure of the current graph rather than just the degree, were much more capable of keeping two and threenode correlation close to time-invariant.

Future work involves analysis of link-based methods and impact of higher-order degree correlation on graph structure.

\section{REFERENCES}

[1] W. Aiello, F. R. K. Chung, and L. Lu, "A Random Graph Model for Massive Graphs," in Proc. ACM STOC, May 2000, pp. 171-180.

[2] R. Albert and A. Barabási, "Topology of Evolving Networks: Local Events and Universality," Phys. Rev. Lett., vol. 85, no. 24, pp. 52345237, Dec. 2000

[3] A. Barabasi, R. Albert, and H. Jeong, "Mean-Field Theory for ScaleFree Random Networks," Physica A, vol. 272, pp. 173-187, 1999.

[4] A.-L. Barabasi and R. Albert, "Emergence of Scaling in Random Networks," Science, vol. 286, no. 5439, pp. 509-512, Oct. 1999.

[5] A. Barrat and R. Pastor-Satorras, "Rate Equation Approach for Correlations in Growing Network Models," Phys. Rev. E, vol. 71, no. 3, Mar. 2005.

[6] E. A. Bender and E. R. Canfield, "The Asymptotic Number of Labeled Graphs with Given Degree Sequences," J. Combin. Theory Ser. A, vol. 24, pp. 296-307, 1978.

[7] G. Bianconi and A. Barabási, "Competition and Multiscaling in Evolving Networks," Europhys. Lett., vol. 54, no. 4, pp. 436-442, May 2001.

[8] M. Boguna and R. Pastor-Satorras, "Class of Correlated Random Networks with Hidden Variables," Phys. Rev. E, vol. 68, no. 3, p. 036112 , Sep. 2003.

[9] B. Bollobás and O. Riordan, "Mathematical Results on Scale-free Random Graphs," in Handbook of Graphs and Networks, S. Bornholdt and H. G. Schuster, Eds. Wiley-VCH, Berlin, 2002.

[10] S. Bornholdt and H. Ebel, "World Wide Web Scaling Exponent from Simon's 1955 Model," Phys. Rev. E, vol. 64, no. 3, p. 035104, Aug. 2001. 
[11] T. Bu and D. Towsley, "On Distinguishing between Internet Power Law Topology Generators," in Proc. IEEE INFOCOM, vol. 2, Jun. 2002, pp. 638-647.

[12] G. Caldarelli, A. Capocci, P. D. L. Rios, and M. A. Munoz, "ScaleFree Networks from Varying Vertex Intrinsic Fitness," Phys. Rev. Lett., vol. 89 , no. 25, p. 258702, Dec. 2002.

[13] H. Chang, S. Jamin, and W. Willinger, "To Peer or not to Peer: Modeling the Evolution of the Internets AS-level Topology," in Proc. IEEE INFOCOM, Apr. 2006.

[14] F. R. K. Chung and L. Lu, "Connected Components in Random Graphs with Given Expected Degree Sequences," Annals of Combinatorics, vol. 6, no. 2, pp. 125-145, Nov. 2002.

[15] S. N. Dorogovtsev, "Clustering of Correlated Networks," Phys. Rev. E, vol. 69, no. 2, p. 027104, Feb. 2004.

[16] S. N. Dorogovtsev and J. F. F. Mendes, Evolution of Networks: From Biological Nets to the Internet and WWW. Oxford Univ. Press, 2003.

[17] P. Erdös and A. Rényi, "On Random Graphs I," Publication Math. Debrecen, vol. 6, pp. 290-297, 1959.

[18] A. Fabrikant, E. Koutsoupias, and C. Papadimitriou, "Heuristically Optimized Tradeoffs," in Proc. ICALP, Jul. 2002, pp. 110-122.

[19] M. Faloutsos, P. Faloutsos, and C. Faloutsos, "On Power-law Relationships of the Internet Topology," in Proc. ACM SIGCOMM, Aug. 1999, pp. 251-262.

[20] K. I. Goh, B. Kahng, and D. Kim, "Universal Behavior of Load Distribution in Scale-Free Networks,” Phys. Rev. Lett., vol. 87, no. 27, p. 278701, Dec. 2001.

[21] Y. He, G. Siganos, M. Faloutsos, and S. V. Krishnamurthy, "A Systematic Framework for Unearthing the Missing Links: Measurements and Impact," in Proc. USENIX/SIGCOMM NSDI, Apr. 2007, pp. 3632-3637.

[22] P. Holme and J. Zhao, "Exploring the Assortativity-Clustering Space of a Network's Degree Sequence," Phys. Rev. E, vol. 75, no. 4, p. 046111, Apr. 2007.

[23] C. Jin, Q. Chen, and S. Jamin, "Inet: Internet Topology," Univ. of Michigan, Tech. Rep. CSE-TR-456-02, 2002.

[24] S. Jin and A. Bestavros, "Small-world Internet Topologies: Possible Causes and Implications on Scalability of End-system Multicast," in Proc. IEEE/ACM International Symposium on Modeling, Analysis and Simulation (MASCOTS), Oct. 2003.

[25] K. Klemm and V. M. Eguiluz, "Growing Scale-Free Networks with Small World Behavior," Phys. Rev. E, vol. 65, no. 5, p. 057102, May 2002.

[26] P. L. Krapivsky, S. Redner, and F. Leyvraz, "Connectivity of Growing Random Networks," Phys. Rev. Lett., vol. 85, no. 21, pp. 4629-4632, Nov. 2000.

[27] T. Luczak, "Sparse Random Graphs with a Given Degree Sequence," Random Graphs, vol. 2, pp. 165-182, 1992.

[28] D. Magoni and J. Pansiot, "Analysis of the Autonomous System Network Topology," ACM SIGCOMM Comp. Comm. Rev., vol. 31, pp. 26-37, 2001.

[29] P. Mahadevan, D. Krioukov, K. Fall, and A. Vahdat, "Systematic Topology Analisys and Generation Using Degree Correlations," in Proc. ACM SIGCOMM, Sep. 2006, pp. 135-146.

[30] M. Mihail and N. Visnoi, "On Generating Graphs with Prescribed Degree Sequences for Complex Network Modeling Applications," in Proc. ARACNE, Sep. 2002.

[31] M. Molloy and B. Reed, "A Critical Point for Random Graphs with a Given Degree Sequence," Random Structures and Algorithms, vol. 6, pp. 161-179, 1995.

[32] M. E. J. Newman, "Assortative Mixing in Networks," Phys. Rev. Lett., vol. 89, no. 20, p. 208701, Oct. 2002.

[33] M. E. J. Newman, "Random Graphs as Models of Networks," in Handbook of Graphs and Networks, S. Bornholdt and H. G. Schuster, Eds. Wiley-VCH, Berlin, 2002.

[34] M. E. J. Newman, "Mixing Patterns in Networks," Phys. Rev. E, vol. 67, no. 2, p. 026126, Feb. 2003.

[35] M. E. J. Newman and J. Park, "Why Social Networks are Different from Other Types of Networks," Phys. Rev. E, vol. 68, no. 3, p. 036122, Sep. 2003.

[36] M. E. J. Newman, S. H. Strogatz, and D. J. Watts, "Random Graphs with Arbitrary Degree Distributions and their Applications," Phys. Rev. E, vol. 64, no. 2, p. 026118, Jul. 2001.

[37] R. Pastor-Satorras, A. Vazquez, and A. Vespignani, "Dynamical and Correlation Properties of the Internet," Phys. Rev. Lett., vol. 87, no. 25, p. 258701, Dec. 2001.

[38] R. Pastor-Satorras and A. Vespignani, Evolution and Structure of the Internet: A Statistical Physics Approach. Cambridge University Press, 2004.
[39] A. R. Puniyani, R. M. Lukose, and B. A. Huberman, "Intentional Walks on Scale Free Small Worlds," cond-mat/0107212, Jul. 2001.

[40] RIPE. [Online]. Available: http://www.ripe.net/.

[41] Route Views Project. [Online]. Available: http://www.antc.uoregon.edu/ route-views.

[42] M. A. Serrano and M. Boguna, "Tuning Clustering in Random Networks with Arbitrary Degree Distributions," Phys. Rev. E, vol. 72, no. 3, p. 036133, Sep. 2005.

[43] H. A. Simon, "On a Class of Skew Distribution Functions," Biometrika, vol. 42, pp. 425-440, 1955.

[44] H. A. Simon, "Some Further Notes on a Class of Skew Distribution Functions," Information and Control, vol. 3, pp. 80-88, 1960.

[45] B. Soderberg, "General Formalism for Inhomogeneous Random Graphs," Phys. Rev. E, vol. 66, no. 6, p. 066121, Dec. 2002.

[46] S. N. Soffer and A. Vazquez, "Network Clustering Coefficient without Degree-Correlation Biases," Phys. Rev. E, vol. 71, no. 5, p. 057101, May 2005.

[47] G. Szabo, M. Alava, and J. Kertesz, "Structural Transitions in Scale-Free Networks," Phys. Rev. E, vol. 67, no. 5, p. 056102, May 2003.

[48] H. Tangmunarunkit, R. Govindan, S. Jamin, S. Shenker, and W. Willinger, "Network Topology Generators: Degree-Based vs. Structural," in Proc. ACM SIGCOMM, Aug. 2002, pp. 147-159.

[49] A. Vazquez, R. Pastor-Satorras, and A. Vespignani, "Large-Scale Topological and Dynamical Properties of the Internet," Phys. Rev. E, vol. 65, no. 6, p. 066130, Jun. 2002.

[50] X. Wang and D. Loguinov, "Wealth-Based Evolution Model for the Internet AS-Level Topology," in Proc. IEEE INFOCOM, Apr. 2006.

[51] D. J. Watts and S. Strogatz, "Collective Dynamics of Small World Networks," Nature, vol. 393, pp. 440-442, Jun. 1998.

[52] S. Yook, H. Jeong, and A. Barabási, "Modeling the Internet's Largescale Topology," Natl. Acad. Sci., vol. 99, no. 21, pp. 13382-13386, Oct. 2002.

[53] S. Zhou and R. J. Mondragon, "Accurately Modeling the Internet Topology," Phys. Rev. E, vol. 70, no. 6, p. 066108, Dec. 2004.

\section{APPENDIX I \\ PROOF OF LEMMA 5}

Proof: In what follows, we first divide the double integral in $\phi(x)$ into three simple components and reduce them to single-integrals, then derive these single integrals for different regions of $x$, and finally identify asymptotically significant terms to prove this theorem.

\section{A. Decomposing $\phi(x)$}

We split function $\phi(x)$ into three integrals:

$$
\begin{aligned}
\phi(x)=\int_{\beta}^{\frac{D}{\beta}} & \int_{\beta}^{\frac{D}{u}} \frac{u v}{D} \pi(x, u) \pi(x, v) f(u) f(v) d v d u \\
& +\int_{\beta}^{\frac{D}{\beta}} \int_{\frac{D}{u}}^{\infty} \pi(x, u) \pi(x, v) f(u) f(v) d v d u \\
& +\int_{\frac{D}{\beta}}^{\infty} \int_{\beta}^{\infty} \pi(x, v) f(u) f(v) d v d u,
\end{aligned}
$$

which we call $\phi_{1}(x), \phi_{2}(x), \phi_{3}(x)$, respectively. We next derive each of them separately.

Since we are only interested in the asymptotics of $\phi(x)$, we next identify the dominant terms in $\phi_{i}(x)$ and then choose the most significant one for predicting $\phi(x)$ in the limit of graph size $n$. For the rest of the paper, we denote by $\theta_{i}(x)$ the leading term in $\phi_{i}(x)$ and by $\theta(x)$ the one in $\phi(x)$.

1) Deriving $\phi_{1}(x)$ : We first formulate the integral associated with variable $v$ and reduce the double integral to a single 
integral. For $\phi_{1}(x)$, we have:

$$
\begin{aligned}
\phi_{1}(x) & =\int_{\beta}^{\frac{D}{\beta}} \int_{\beta}^{\frac{D}{u}} \frac{u v}{D} \pi(x, u) \pi(x, v) f(u) f(v) d v d u \\
& =\int_{\beta}^{\frac{D}{\beta}} \frac{u}{D} \pi(x, u) f(u) \int_{\beta}^{\frac{D}{u}} v \pi(x, v) f(v) d v d u .
\end{aligned}
$$

To simply $\phi_{1}(x)$ and focus on the inner integral with $v$, we define $g(u)$ to be:

$$
g(u)=\frac{u}{D} \pi(x, u) f(u)
$$

and then reduce $\phi_{1}(x)$ to:

$$
\phi_{1}(x)=\int_{\beta}^{\frac{D}{\beta}} g(u) \int_{\beta}^{\frac{D}{u}} v \pi(x, v) f(v) d v d u .
$$

Further breaking down the outside integral of $\phi_{1}(x)$ into the following two parts: $\phi_{11}(x)$ and $\phi_{12}(x)$ for $\left[x, \frac{D}{\beta}\right]$ and $[\beta, x]$, respectively. Now, $\phi_{1}(x)=\phi_{11}(x)+\phi_{12}(x)$ with:

$$
\begin{aligned}
\phi_{11}(x) & =\int_{x}^{\frac{D}{\beta}} g(u) \int_{\beta}^{\frac{D}{u}} v \pi(x, v) f(v) d v d u, \\
\phi_{12}(x) & =\int_{\beta}^{x} g(u) \int_{\beta}^{\frac{D}{u}} v \pi(x, v) f(v) d v d u,
\end{aligned}
$$

which we will solve next.

We first examine $\phi_{11}(x)$, for which $x \leq u$ and $v \leq \frac{D}{u}$. It follows that $v x \leq D$ and $\pi(x, v)=\min \left(\frac{v x}{D}, 1\right)=\frac{v x}{D}$, which establishes that:

$$
\phi_{11}(x)=\int_{x}^{\frac{D}{\beta}} g(u) \int_{\beta}^{\frac{D}{u}} \frac{x v^{2}}{D} f(v) d v d u .
$$

Substituting $f(v)=\alpha \beta^{\alpha} v^{-\alpha-1}$ into (112) and solving the inside integral, we get the following equality:

$$
\begin{aligned}
\phi_{11}(x)= & \int_{x}^{\frac{D}{\beta}} g(u) \frac{x \alpha \beta^{\alpha}}{D(2-\alpha)}\left(\left(\frac{D}{u}\right)^{2-\alpha}-\beta^{2-\alpha}\right) d u \\
= & \frac{x \alpha \beta^{\alpha}}{D^{\alpha-1}(2-\alpha)} \int_{x}^{\frac{D}{\beta}} g(u) u^{\alpha-2} d u \\
& +\frac{x \alpha \beta^{2}}{D(2-\alpha)} \int_{x}^{\frac{D}{\beta}} g(u) d u .
\end{aligned}
$$

To simplify $\phi_{11}$, define $b_{1}(x)$ and $b_{2}(x)$ to be:

$$
b_{1}(x)=\int_{x}^{\frac{D}{\beta}} g(u) u^{\alpha-2} d u, \quad b_{2}(x)=\int_{x}^{\frac{D}{\beta}} g(u) d u,
$$

from which $\phi_{11}$ becomes:

$$
\phi_{11}(x)=\frac{x \alpha \beta^{\alpha}}{D^{\alpha-1}(2-\alpha)} b_{1}(x)+\frac{x \alpha \beta^{2}}{D(2-\alpha)} b_{2}(x) .
$$

We defer the derivations of $b_{1}(x)$ and $b_{2}(x)$ to the later part of this proof and proceed to formulate $\phi_{12}(x)$. We split the inner integral in $\phi_{12}(x)$ into two parts for $v<\frac{D}{x}$ and $v \geq \frac{D}{x}$, respectively. It follows that:

$$
\begin{aligned}
\phi_{12}(x)= & \int_{\beta}^{x} g(u) \int_{\beta}^{\frac{D}{x}} v \pi(x, v) f(v) d v d u \\
& +\int_{\beta}^{x} g(u) \int_{\frac{D}{x}}^{\frac{D}{u}} v \pi(x, v) f(v) d v d u .
\end{aligned}
$$

Since $\pi(x, v)$ can be determined separately for the two terms in $\phi_{12}(x)$ :

$$
\pi(x, v)= \begin{cases}\frac{x v}{D} & v<\frac{D}{x} \\ 1 & v \geq \frac{D}{x}\end{cases}
$$

it follows that:

$$
\begin{aligned}
\phi_{12}(x)= & \int_{\beta}^{x} g(u) \int_{\beta}^{\frac{D}{x}} \frac{x v^{2}}{D} f(v) d v d u \\
& +\int_{\beta}^{x} g(u) \int_{\frac{D}{x}}^{\frac{D}{u}} v f(v) d v d u .
\end{aligned}
$$

Solving the inside integral of (118), we obtain:

$$
\begin{aligned}
\phi_{12}(x)= & \int_{\beta}^{x} g(u) \frac{x \alpha \beta^{\alpha}}{D(2-\alpha)}\left(\left(\frac{D}{x}\right)^{2-\alpha}-\beta^{2-\alpha}\right) d u \\
& +\int_{\beta}^{x} g(u) \frac{\alpha \beta^{\alpha}}{1-\alpha}\left(\left(\frac{D}{u}\right)^{1-\alpha}-\left(\frac{D}{x}\right)^{1-\alpha}\right) d u \\
= & \left(\frac{-\alpha \beta^{\alpha}}{(2-\alpha)(1-\alpha)}\left(\frac{D}{x}\right)^{1-\alpha}-\frac{x \alpha \beta^{2}}{D(2-\alpha)}\right) \int_{\beta}^{x} g(u) d u \\
& +\frac{\alpha \beta^{\alpha}}{D^{\alpha-1}(1-\alpha)} \int_{\beta}^{x} g(u) u^{\alpha-1} d u
\end{aligned}
$$

For simplicity, define $b_{3}(x)$ and $b_{4}(x)$ to be:

$$
b_{3}(x)=\int_{\beta}^{x} g(u) d u, \quad b_{4}(x)=\int_{\beta}^{x} g(u) u^{\alpha-1} d u,
$$

from which it follows that:

$$
\begin{aligned}
\phi_{12}(x)= & \left(\frac{-\alpha \beta^{\alpha}}{(2-\alpha)(1-\alpha)}\left(\frac{D}{x}\right)^{1-\alpha}-\frac{x \alpha \beta^{2}}{D(2-\alpha)}\right) b_{3}(x) \\
& +\frac{\alpha \beta^{\alpha}}{D^{\alpha-1}(1-\alpha)} b_{4}(x) .
\end{aligned}
$$

Again, we leave the derivations of $b_{3}(x)$ and $b_{4}(x)$ to the next subsection and next examine $\phi_{2}(x)$.

2) Deriving $\phi_{2}(x)$ : Using a similar method as before, we obtain the following formula for $\phi_{2}(x)$ :

$$
\begin{aligned}
\phi_{2}(x) & =\int_{\beta}^{\frac{D}{\beta}} \int_{\frac{D}{u}}^{\infty} \pi(x, u) \pi(x, v) f(u) f(v) d v d u \\
& =\int_{\beta}^{\frac{D}{\beta}} \pi(x, u) f(u) \int_{\frac{D}{u}}^{\infty} \pi(x, v) f(v) d v d u .
\end{aligned}
$$

Again, we define $h(u)$ to be:

$$
h(u)=\pi(x, u) f(u),
$$

and reduce $\phi_{2}(x)$ to become:

$$
\phi_{2}(x)=\int_{\beta}^{\frac{D}{\beta}} h(u) \int_{\frac{D}{u}}^{\infty} \pi(x, v) f(v) d v d u .
$$

Notice that we need the exact forms of function $\pi(.,$. to solve the double integral. Therefore, we further split the 
integral into the following three parts, $\phi_{21}(x), \phi_{22}(x), \phi_{23}(x)$, respectively, where $\pi(.,$.$) can be easily determined:$

$$
\begin{aligned}
\phi_{2}(x)= & \int_{\beta}^{x} h(u) \int_{\frac{D}{u}}^{\infty} \pi(x, v) f(v) d v d u \\
& +\int_{x}^{\frac{D}{\beta}} h(u) \int_{\frac{D}{u}}^{\frac{D}{x}} \pi(x, v) f(v) d v d u \\
& +\int_{x}^{\frac{D}{\beta}} h(u) \int_{\frac{D}{x}}^{\infty} \pi(x, v) f(v) d v d u .
\end{aligned}
$$

Noticing that $x>u$ and $v>\frac{D}{u}$ in $\phi_{21}(x)$, we thus establish that $\pi(x, v)=1$ and reduce $\phi_{21}(x)$ to:

$$
\phi_{21}(x)=\int_{\beta}^{x} h(u) \int_{\frac{D}{u}}^{\infty} f(v) d v d u .
$$

Solving the inside integral in $\phi_{21}(x)$ establishes that:

$$
\begin{aligned}
\phi_{21}(x) & =\int_{\beta}^{x} h(u) \beta^{\alpha}\left(\frac{D}{u}\right)^{-\alpha} d u \\
& =\frac{\beta^{\alpha}}{D^{\alpha}} \int_{\beta}^{x} h(u) u^{\alpha} d u .
\end{aligned}
$$

Defining $b_{5}(x)$ to be:

$$
b_{5}(x)=\int_{\beta}^{x} h(u) u^{\alpha} d u,
$$

we obtain the following equality:

$$
\phi_{21}(x)=\frac{\beta^{\alpha}}{D^{\alpha}} b_{5}(x) .
$$

Now, we start to examine $\phi_{22}(x)$. Since $v \leq \frac{D}{x}$, we have $\pi(x, v)=\frac{x v}{D}$ and thus $\phi_{22}(x)$ becomes:

$$
\begin{aligned}
\phi_{22}(x) & =\int_{x}^{\frac{D}{\beta}} h(u) \int_{\frac{D}{u}}^{\frac{D}{x}} \frac{x v}{D} f(v) d v d u \\
& =\int_{x}^{\frac{D}{\beta}} h(u) \frac{x \alpha \beta^{\alpha}}{D} \int_{\frac{D}{u}}^{\frac{D}{x}} v^{-\alpha} d v d u .
\end{aligned}
$$

Solving the inside integral in $\phi_{22}(x)$ gives:

$$
\begin{aligned}
\phi_{22}(x)= & \int_{x}^{\frac{D}{\beta}} h(u) \frac{x \alpha \beta^{\alpha}}{D(1-\alpha)}\left(\left(\frac{D}{x}\right)^{1-\alpha}-\left(\frac{D}{u}\right)^{1-\alpha}\right) d u \\
= & \frac{x^{\alpha} \alpha \beta^{\alpha}}{D^{\alpha}(1-\alpha)} \int_{x}^{\frac{D}{\beta}} h(u) d u \\
& -\frac{x \alpha \beta^{\alpha}}{D^{\alpha}(1-\alpha)} \int_{x}^{\frac{D}{\beta}} h(u) u^{\alpha-1} d u .
\end{aligned}
$$

Define $b_{6}(x)$ and $b_{7}(x)$ to be:

$$
b_{6}(x)=\int_{x}^{\frac{D}{\beta}} h(u) d u, \quad b_{7}(x)=\int_{x}^{\frac{D}{\beta}} h(u) u^{\alpha-1} d u,
$$

from which $\phi_{22}(x)$ becomes:

$$
\phi_{22}(x)=\frac{x^{\alpha} \alpha \beta^{\alpha}}{D^{\alpha}(1-\alpha)} b_{6}(x)-\frac{x \alpha \beta^{\alpha}}{D^{\alpha}(1-\alpha)} b_{7}(x) .
$$

Next, we tackle the last element in computing $\phi_{2}(x)$. Consider that for $v \leq \frac{D}{x}$, we have $\pi(x, v)=1$ in $\phi_{23}(x)$. Therefore, it is easy to verify that:

$$
\phi_{23}(x)=\int_{x}^{\frac{D}{\beta}} h(u) \int_{\frac{D}{x}}^{\infty} f(v) d v d u .
$$

Solving the inside integral of $\phi_{23}(x)$ leads to:

$$
\begin{aligned}
\phi_{23}(x) & =\int_{x}^{\frac{D}{\beta}} h(u) \beta^{\alpha}\left(\frac{D}{x}\right)^{-\alpha} d u \\
& =\frac{x^{\alpha} \beta^{\alpha}}{D^{\alpha}} \int_{x}^{\frac{D}{\beta}} h(u) d u=\frac{x^{\alpha} \beta^{\alpha}}{D^{\alpha}} b_{6}(x),
\end{aligned}
$$

where $b_{6}(x)$ is given in (132).

3) Deriving $\phi_{3}(x)$ : It is fairly straightforward to derive $\phi_{3}(x)$ by first deriving the integral associated with variable $u$ :

$$
\phi_{3}(x)=\frac{\alpha \beta^{2 \alpha}}{D^{\alpha}} \int_{\beta}^{\infty} \pi(x, v) \alpha \beta^{\alpha} v^{-\alpha-1} d v .
$$

Further splitting the integral in $\phi_{3}(x)$ into $\left[\beta, \frac{D}{x}\right]$ and $\left[\frac{D}{x}, \infty\right)$ establishes:

$$
\begin{aligned}
\phi_{3}(x)= & \frac{\alpha^{2} \beta^{3 \alpha}}{D^{\alpha}} \int_{\beta}^{\frac{D}{x}} \frac{x v}{D} v^{-\alpha-1} d v+\frac{\alpha^{2} \beta^{3 \alpha}}{D^{\alpha}} \int_{\frac{D}{x}}^{\infty} v^{-\alpha-1} d v \\
= & \frac{x \alpha^{2} \beta^{3 \alpha}}{D^{\alpha+1}(1-\alpha)}\left(\left(\frac{D}{x}\right)^{1-\alpha}-\beta^{1-\alpha}\right) \\
& +\frac{\alpha \beta^{3 \alpha}}{D^{\alpha}}\left(\frac{D}{x}\right)^{-\alpha} .
\end{aligned}
$$

With the above results, we are ready to expand $\phi(x)$ for its asymptotic formula.

\section{B. Assembling $\phi(x)$ for $x \in[\beta, \sqrt{D})$ and $\alpha \in(1,2)$}

In what follows, we first derive $b_{1}(x)-b_{7}(x)$ and then develop the asymptotic form of $\phi(x)$ by identifying significant terms in $\phi_{1}(x)-\phi_{3}(x)$.

1) Deriving $b_{i}(x)$ : Next, we expand the integrals $\left\{b_{i}(x)\right\}$ for $i=1 \ldots 7$ with $x \in[\beta, \sqrt{D})$ and $\alpha<2$.

Since $x \in[\beta, \sqrt{D})$, it follows that $x$ is smaller than $\frac{D}{x}$ and thus the interval $\left[x, \frac{D}{\beta}\right)$ could be split into two parts: $\left[x, \frac{D}{x}\right)$ and $\left[\frac{D}{x}, \frac{D}{\beta}\right)$, where $\pi(x, u)=\frac{x u}{D}$ and 1 , respectively. Therefore, we replace the corresponding values of $\pi(x, u)$ in $b_{1}(x)$ to establish:

$$
\begin{aligned}
b_{1}(x) & =\int_{x}^{\frac{D}{\beta}} \frac{u}{D} \pi(x, u) f(u) u^{\alpha-2} d u \\
& =\int_{x}^{\frac{D}{x}} \frac{u}{D} \frac{x u}{D} f(u) u^{\alpha-2} d u+\int_{\frac{D}{x}}^{\frac{D}{\beta}} \frac{u}{D} f(u) u^{\alpha-2} d u \\
& =\frac{x \alpha \beta^{\alpha}}{D^{2}} \int_{x}^{\frac{D}{x}} u^{-1} d u+\frac{\alpha \beta^{\alpha}}{D} \int_{\frac{D}{x}}^{\frac{D}{\beta}} u^{-2} d u .
\end{aligned}
$$

Further expanding the integral in $b_{1}(x)$ leads to:

$$
\begin{aligned}
b_{1}(x) & =\frac{x \alpha \beta^{\alpha}}{D^{2}}(\log D-2 \log x)-\frac{\alpha \beta^{\alpha}}{D}\left(\frac{\beta}{D}-\frac{x}{D}\right) \\
& =\frac{x \alpha \beta^{\alpha}}{D^{2}}(\log D-2 \log x)-\frac{\alpha \beta^{\alpha+1}}{D^{2}}+\frac{x \alpha \beta^{\alpha}}{D^{2}} .
\end{aligned}
$$


Similarly, we split the integral in $b_{2}(x)$ as for $b_{1}(x)$ and obtain the following formula:

$$
\begin{aligned}
b_{2}(x) & =\int_{x}^{\frac{D}{\beta}} \frac{u}{D} \pi(x, u) f(u) d u \\
& =\int_{x}^{\frac{D}{x}} \frac{u}{D} \frac{x u}{D} f(u) d u+\int_{\frac{D}{x}}^{\frac{D}{\beta}} \frac{u}{D} f(u) d u \\
& =\frac{x \alpha \beta^{\alpha}}{D^{2}} \int_{x}^{\frac{D}{x}} u^{1-\alpha} d u+\frac{\alpha \beta^{\alpha}}{D} \int_{\frac{D}{x}}^{\frac{D}{\beta}} u^{-\alpha} d u .
\end{aligned}
$$

Further solving the integral in $b_{2}(x)$, we establish that:

$$
\begin{aligned}
b_{2}(x)= & \frac{x \alpha \beta^{\alpha}}{D^{2}(2-\alpha)}\left(\left(\frac{D}{x}\right)^{2-\alpha}-x^{2-\alpha}\right) \\
& +\frac{\alpha \beta^{\alpha}}{D^{\alpha}(1-\alpha)}\left(\beta^{\alpha-1}-x^{\alpha-1}\right) \\
= & \frac{x^{\alpha-1} \alpha \beta^{\alpha}}{D^{\alpha}(2-\alpha)}-\frac{x^{3-\alpha} \alpha \beta^{\alpha}}{D^{2}(2-\alpha)} \\
& +\frac{\alpha \beta^{2 \alpha-1}}{D^{\alpha}(1-\alpha)}-\frac{x^{\alpha-1} \alpha \beta^{\alpha}}{D^{\alpha}(1-\alpha)},
\end{aligned}
$$

It is straightforward to derive $b_{3}(x)-b_{5}(x)$ in that for their integral ranges $[\beta, x]$, link existence function $\pi(x, u)$ equals to $\frac{x u}{D}$. It follows that:

$$
\begin{aligned}
b_{3}(x) & =\int_{\beta}^{x} \frac{u}{D} \pi(x, u) f(u) d u=\int_{\beta}^{x} \frac{u}{D} \frac{x u}{D} f(u) d u \\
& =\frac{x \alpha \beta^{\alpha}}{D^{2}} \int_{\beta}^{x} u^{1-\alpha} d u \\
& =\frac{x^{3-\alpha} \alpha \beta^{\alpha}}{D^{2}(2-\alpha)}-\frac{x \alpha \beta^{2}}{D^{2}(2-\alpha)}, \\
b_{4}(x) & =\int_{\beta}^{x} \frac{u}{D} \pi(x, u) f(u) u^{\alpha-1} d u \\
& =\int_{\beta}^{x} \frac{u}{D} \frac{x u}{D} f(u) u^{\alpha-1} d u \\
& =\frac{x \alpha \beta^{\alpha}}{D^{2}}(x-\beta)=\frac{x^{2} \alpha \beta^{\alpha}}{D^{2}}-\frac{x \alpha \beta^{\alpha+1}}{D^{2}}, \\
b_{5}(x) & =\int_{\beta}^{x} \pi(x, u) f(u) u^{\alpha} d u=\int_{\beta}^{x} \frac{x u}{D} f(u) u^{\alpha} d u \\
& =\frac{x \alpha \beta^{\alpha}}{D}(x-\beta)=\frac{x^{2} \alpha \beta^{\alpha}}{D}-\frac{x \alpha \beta^{\alpha+1}}{D} .
\end{aligned}
$$

The derivations of $b_{6}(x)$ and $b_{7}(x)$ are similar to that of $b_{1}(x)$ and require to split the integral range $\left[x, \frac{D}{\beta}\right]$ into $\left[x, \frac{D}{x}\right]$ and $\left[\frac{D}{x}, \frac{D}{\beta}\right]$. For $b_{6}(x)$, we substitute the corresponding values of $\pi(x, u)$ into $b_{6}(x)$ and obtain the following equality:

$$
\begin{aligned}
b_{6}(x) & =\int_{x}^{\frac{D}{\beta}} \pi(x, u) f(u) d u \\
& =\int_{x}^{\frac{D}{x}} \frac{x u}{D} f(u) d u+\int_{\frac{D}{x}}^{\frac{D}{\beta}} f(u) d u \\
& =\frac{x \alpha \beta^{\alpha}}{D} \int_{x}^{\frac{D}{x}} u^{-\alpha} d u+\alpha \beta^{\alpha} \int_{\frac{D}{x}}^{\frac{D}{\beta}} u^{-\alpha-1} d u .
\end{aligned}
$$

Further expanding the integral in $b_{6}(x)$ establishes:

$$
\begin{aligned}
b_{6}(x)= & \frac{x \alpha \beta^{\alpha}}{D(1-\alpha)}\left(\left(\frac{D}{x}\right)^{1-\alpha}-x^{1-\alpha}\right) \\
& +\beta^{\alpha}\left(\left(\frac{D}{\beta}\right)^{-\alpha}-\left(\frac{D}{x}\right)^{-\alpha}\right) \\
= & \frac{x^{\alpha} \alpha \beta^{\alpha}}{D^{\alpha}(1-\alpha)}-\frac{x^{2-\alpha} \alpha \beta^{\alpha}}{D(1-\alpha)}+\frac{\beta^{2 \alpha}}{D^{\alpha}}-\frac{x^{\alpha} \beta^{\alpha}}{D^{\alpha}} .
\end{aligned}
$$

Similarly, we obtain $b_{7}(x)$ as follows:

$$
\begin{aligned}
b_{7}(x) & =\int_{x}^{\frac{D}{\beta}} \pi(x, u) f(u) u^{\alpha-1} d u \\
& =\int_{x}^{\frac{D}{x}} \frac{x u}{D} f(u) u^{\alpha-1} d u+\int_{\frac{D}{x}}^{\frac{D}{\beta}} f(u) u^{\alpha-1} d u \\
& =\frac{x \alpha \beta^{\alpha}}{D} \int_{x}^{\frac{D}{x}} u^{-1} d u+\alpha \beta^{\alpha} \int_{\frac{D}{x}}^{\frac{D}{\beta}} u^{-2} d u \\
& =\frac{x \alpha \beta^{\alpha}(\log D-2 \log x)}{D}+\frac{\alpha \beta^{\alpha}(x-\beta)}{D} .
\end{aligned}
$$

With the results of $b_{1}(x)-b_{7}(x)$ in (138)-(146), we are ready to derive $\phi_{1}(x)-\phi_{3}(x)$, which we will perform in what follows.

2) Deriving $\phi_{i}(x)$ : First, we focus on $\phi_{1}(x)$. Substituting (138)-(140) into $\phi_{11}(x)$ in (115), it follows that:

$$
\begin{aligned}
\phi_{11}(x)= & \frac{x \alpha \beta^{\alpha}}{D^{\alpha-1}(2-\alpha)}\left(\frac{x \alpha \beta^{\alpha}}{D^{2}}(\log D-2 \log x)\right. \\
& \left.-\frac{\alpha \beta^{\alpha+1}}{D^{2}}+\frac{x \alpha \beta^{\alpha}}{D^{2}}\right) \\
& +\frac{x \alpha \beta^{2}}{D(2-\alpha)}\left(\frac{x^{\alpha-1} \alpha \beta^{\alpha}}{D^{\alpha}(2-\alpha)}-\frac{x^{3-\alpha} \alpha \beta^{\alpha}}{D^{2}(2-\alpha)}\right. \\
& \left.+\frac{\alpha \beta^{2 \alpha-1}}{D^{\alpha}(1-\alpha)}-\frac{x^{\alpha-1} \alpha \beta^{\alpha}}{D^{\alpha}(1-\alpha)}\right)
\end{aligned}
$$

and (141)-(142), $\phi_{12}(x)$ in (121) reduces to:

$$
\begin{aligned}
\phi_{12}(x)= & \left(\frac{-\alpha \beta^{\alpha}\left(\frac{D}{x}\right)^{1-\alpha}}{(2-\alpha)(1-\alpha)}-\frac{x \alpha \beta^{2}}{D(2-\alpha)}\right) \frac{x^{3-\alpha} \alpha \beta^{\alpha}-x \alpha \beta^{2}}{D^{2}(2-\alpha)} \\
& +\frac{\alpha \beta^{\alpha}}{D^{\alpha-1}(1-\alpha)} \frac{x^{2} \alpha \beta^{\alpha}-x \alpha \beta^{\alpha+1}}{D^{2}}
\end{aligned}
$$

Then, it follows from (147) and (148) that $\phi_{1}(x)$ becomes:

$$
\begin{aligned}
\phi_{1}(x)= & \phi_{11}(x)+\phi_{12}(x) \\
= & \frac{x^{2} \alpha^{2} \beta^{2 \alpha}}{D^{\alpha+1}(2-\alpha)}(\log D-2 \log x)-\frac{x \alpha^{2} \beta^{2 \alpha+1}}{D^{\alpha+1}(2-\alpha)} \\
& +\frac{x^{2} \alpha^{2} \beta^{2 \alpha}}{D^{\alpha+1}(2-\alpha)}+\frac{x^{\alpha} \alpha^{2} \beta^{\alpha+2}}{D^{\alpha+1}(2-\alpha)^{2}}-\frac{x^{4-\alpha} \alpha^{2} \beta^{\alpha+2}}{D^{3}(2-\alpha)^{2}} \\
& +\frac{x \alpha^{2} \beta^{2 \alpha+1}}{D^{\alpha+1}(2-\alpha)(1-\alpha)}-\frac{x^{\alpha} \alpha^{2} \beta^{\alpha+2}}{D^{\alpha+1}(2-\alpha)(1-\alpha)} \\
& -\frac{x^{2} \alpha^{2} \beta^{2 \alpha}}{D^{\alpha+1}(2-\alpha)^{2}(1-\alpha)}+\frac{x^{\alpha} \alpha^{2} \beta^{\alpha+2}}{D^{\alpha+1}(2-\alpha)^{2}(1-\alpha)} \\
& -\frac{x^{4-\alpha} \alpha^{2} \beta^{\alpha+2}}{D^{3}(2-\alpha)^{2}}+\frac{x^{2} \alpha^{2} \beta^{4}}{D^{3}(2-\alpha)^{2}} .
\end{aligned}
$$


Next, we start to derive $\phi_{2}(x)$. Substituting (143) into $\phi_{21}(x)$ in (129), it follows that:

$$
\begin{aligned}
\phi_{21}(x)= & \frac{\beta^{\alpha}}{D^{\alpha}}\left(\frac{x^{2} \alpha \beta^{\alpha}}{D}-\frac{x \alpha \beta^{\alpha+1}}{D}\right) \\
= & \frac{x^{2} \alpha \beta^{2 \alpha}}{D^{\alpha+1}}-\frac{x \alpha \beta^{2 \alpha+1}}{D^{\alpha+1}} .
\end{aligned}
$$

Similarly, the following equations holds for $\phi_{22}(x)$ by substituting (145)-(146) into (133):

$$
\begin{aligned}
\phi_{22}(x)= & \frac{x^{\alpha} \alpha \beta^{\alpha}}{D^{\alpha}(1-\alpha)}\left(\frac{x^{\alpha} \alpha \beta^{\alpha}}{D^{\alpha}(1-\alpha)}-\frac{x^{2-\alpha} \alpha \beta^{\alpha}}{D(1-\alpha)}\right. \\
& \left.+\frac{\beta^{2 \alpha}-x^{\alpha} \beta^{\alpha}}{D^{\alpha}}\right)-\frac{x \alpha \beta^{\alpha}}{D^{\alpha}(1-\alpha)} \\
& \times \frac{x \alpha \beta^{\alpha}(\log D-2 \log x)+\alpha \beta^{\alpha}(x-\beta)}{D} \\
= & \frac{x^{2 \alpha} \alpha^{2} \beta^{2 \alpha}}{D^{2 \alpha}(1-\alpha)^{2}}-\frac{x^{2} \alpha^{2} \beta^{2 \alpha}}{D^{\alpha+1}(1-\alpha)^{2}}+\frac{x^{\alpha} \alpha \beta^{3 \alpha}}{D^{2 \alpha}(1-\alpha)} \\
& -\frac{x^{2 \alpha} \alpha \beta^{2 \alpha}}{D^{2 \alpha}(1-\alpha)}-\frac{x^{2} \alpha^{2} \beta^{2 \alpha}(\log D-2 \log x)}{D^{\alpha+1}(1-\alpha)} \\
& -\frac{x \alpha^{2} \beta^{2 \alpha}(x-\beta)}{D^{\alpha+1}(1-\alpha)},
\end{aligned}
$$

and for $\phi_{23}(x)$ by combining (135) and (145):

$$
\begin{aligned}
\phi_{23}(x)= & \frac{x^{\alpha} \beta^{\alpha}}{D^{\alpha}}\left(\frac{x^{\alpha} \alpha \beta^{\alpha}}{D^{\alpha}(1-\alpha)}-\frac{x^{2-\alpha} \alpha \beta^{\alpha}}{D(1-\alpha)}+\frac{\beta^{2 \alpha}}{D^{\alpha}}-\frac{x^{\alpha} \beta^{\alpha}}{D^{\alpha}}\right) \\
= & \frac{x^{2 \alpha} \alpha \beta^{2 \alpha}}{D^{2 \alpha}(1-\alpha)}-\frac{x^{2} \alpha \beta^{2 \alpha}}{D^{\alpha+1}(1-\alpha)} \\
& +\frac{x^{\alpha} \beta^{3 \alpha}}{D^{2 \alpha}}-\frac{x^{2 \alpha} \beta^{2 \alpha}}{D^{2 \alpha}} .
\end{aligned}
$$

It follows from (150)-(152) that $\phi_{2}(x)$ is given by:

$$
\begin{aligned}
\phi_{2}(x) & =\phi_{21}(x)+\phi_{22}(x)+\phi_{23}(x) \\
& =\frac{x^{2} \alpha \beta^{2 \alpha}}{D^{\alpha+1}}-\frac{x \alpha \beta^{2 \alpha+1}}{D^{\alpha+1}}+\frac{x^{2 \alpha} \alpha^{2} \beta^{2 \alpha}}{D^{2 \alpha}(1-\alpha)^{2}} \\
& -\frac{x^{2} \alpha^{2} \beta^{2 \alpha}}{D^{\alpha+1}(1-\alpha)^{2}}+\frac{x^{\alpha} \alpha \beta^{3 \alpha}}{D^{2 \alpha}(1-\alpha)}-\frac{x^{2 \alpha} \alpha \beta^{2 \alpha}}{D^{2 \alpha}(1-\alpha)} \\
& -\frac{x^{2} \alpha^{2} \beta^{2 \alpha}(\log D-2 \log x)}{D^{\alpha+1}(1-\alpha)}-\frac{x \alpha^{2} \beta^{2 \alpha}(x-\beta)}{D^{\alpha+1}(1-\alpha)} \\
& +\frac{x^{2 \alpha} \alpha \beta^{2 \alpha}}{D^{2 \alpha}(1-\alpha)}-\frac{x^{2} \alpha \beta^{2 \alpha}}{D^{\alpha+1}(1-\alpha)} \\
& +\frac{x^{\alpha} \beta^{3 \alpha}}{D^{2 \alpha}}-\frac{x^{2 \alpha} \beta^{2 \alpha}}{D^{2 \alpha}} .
\end{aligned}
$$

The integral in $\phi_{3}(x)$ is straightforward and can be simplified as:

$$
\phi_{3}(x)=\frac{x^{\alpha} \alpha \beta^{3 \alpha}}{D^{2 \alpha}(1-\alpha)}-\frac{x \alpha^{2} \beta^{2 \alpha+1}}{D^{\alpha+1}(1-\alpha)} .
$$

Combining $\phi_{1}(x)-\phi_{3}(x)$, we obtain the exact formula for $\phi(x)$. Notice that the final expression of $\phi(x)$ will contain more than 20 summation items, which is not suitable for further asymptotic analysis. It is thus more desirable to omit those non-significant terms from $\phi(x)$, which will be carried out in what follows.
3) Identifying Significant Terms: Since we only consider in this proof that $\alpha \in(1,2)$, we thus can compare the relative significance of summation terms in $\phi(x)$ and establish that the leading terms in $\phi_{1}(x), \phi_{2}(x)$, and $\phi_{3}(x)$ are given by:

$$
\begin{gathered}
\theta_{1}(x)=\frac{x^{2} \alpha^{2} \beta^{2 \alpha} \log D}{D^{\alpha+1}(2-\alpha)}, \quad \theta_{2}(x)=\frac{x^{2} \alpha^{2} \beta^{2 \alpha} \log D}{D^{\alpha+1}(\alpha-1)} \\
\theta_{3}(x)=\frac{x \alpha^{2} \beta^{2 \alpha+1}}{D^{\alpha+1}(\alpha-1)}
\end{gathered}
$$

Notice that $\theta_{1}(x)$ and $\theta_{2}(x)$ have the same asymptotic trends and dominate in $\phi(x)$. Therefore, the leading term $\theta(x)$ in $\phi(x)$ is as follows:

$$
\theta(x)=\theta_{1}(x)+\theta_{2}(x)=\frac{x^{2} \alpha^{2} \beta^{2 \alpha} \log D}{D^{\alpha+1}(2-\alpha)(\alpha-1)} .
$$

Further considering that $D$ is the total expected degrees of the graph, i.e., $D=n E[d]=n \alpha \beta /(\alpha-1)$, we reduce $\theta(x)$ to:

$$
\theta(x)=\Theta\left(x^{2} n^{-\alpha-1} \log n\right),
$$

which establishes the first line in (38).

C. Assembling $\phi(x)$ for $x \in\left[\sqrt{D}, \frac{D}{\beta}\right)$ and $\alpha \in(1,2)$

1) Deriving $b_{i}(x)$ : We first consider the integral range $\left[x, \frac{D}{\beta}\right]$ of $b_{1}(x)$ and $b_{2}(x)$. For $x \in\left[\sqrt{D}, \frac{D}{\beta}\right)$, it follows that $x$ is no less than $\frac{D}{x}$, which establishes that $\pi(x, u)=1$ for $u \in\left[x, \frac{D}{\beta}\right]$. Therefore, $b_{1}(x)$ and $b_{2}(x)$ become:

$$
\begin{gathered}
b_{1}(x)=\frac{\alpha \beta^{\alpha}}{D} \int_{x}^{\frac{D}{\beta}} u^{-2} d u=-\frac{\alpha \beta^{\alpha+1}}{D^{2}}+\frac{\alpha \beta^{\alpha}}{x D} \\
b_{2}(x)=\frac{\alpha \beta^{\alpha}}{D} \int_{x}^{\frac{D}{\beta}} u^{-\alpha} d u=\frac{\alpha \beta^{2 \alpha-1}}{D^{\alpha}(1-\alpha)}-\frac{\alpha \beta^{\alpha} x^{1-\alpha}}{D(1-\alpha)} .
\end{gathered}
$$

Now, we examine the integral range $[\beta, x]$ of $b_{3}(x)-b_{5}(x)$ Noticing that $x$ is no greater than $\frac{D}{x}$, one thus may split the interval $[\beta, x]$ into $\left[\beta, \frac{D}{x}\right]$ and $\left[\frac{D}{x}, x\right]$, where $\pi(x, u)=\frac{x u}{D}$ and 1 , respectively. Therefore, we can replace $\pi(x, u)$ with its corresponding values in $b_{3}(x)-b_{5}(x)$ and then solve the integral. For $b_{3}(x)$, we obtain that:

$$
\begin{aligned}
b_{3}(x) & =\int_{\beta}^{\frac{D}{x}} \frac{u}{D} \frac{x u}{D} f(u) d u+\int_{\frac{D}{x}}^{x} \frac{u}{D} f(u) d u \\
& =\frac{x \alpha \beta^{\alpha}}{D^{2}} \int_{\beta}^{\frac{D}{x}} u^{1-\alpha} d u+\frac{\alpha \beta^{\alpha}}{D} \int_{\frac{D}{x}}^{x} u^{-\alpha} d u .
\end{aligned}
$$

Further expanding the integral in $b_{3}(x)$ results in:

$$
\begin{aligned}
b_{3}(x)= & \frac{x \alpha \beta^{\alpha}}{D^{2}(2-\alpha)}\left(\left(\frac{D}{x}\right)^{2-\alpha}-\beta^{2-\alpha}\right) \\
& +\frac{\alpha \beta^{\alpha}}{D(1-\alpha)}\left(x^{1-\alpha}-\left(\frac{D}{x}\right)^{1-\alpha}\right) \\
= & \frac{x^{\alpha-1} \alpha \beta^{\alpha}}{D^{\alpha}(2-\alpha)(\alpha-1)}-\frac{x \alpha \beta^{2}}{D^{2}(2-\alpha)} \\
& +\frac{x^{1-\alpha} \alpha \beta^{\alpha}}{D(1-\alpha)} .
\end{aligned}
$$


For $b_{4}(x)$, we establish that:

$$
\begin{aligned}
b_{4}(x) & =\int_{\beta}^{\frac{D}{x}} \frac{u}{D} \frac{x u}{D} f(u) u^{\alpha-1} d u+\int_{\frac{D}{x}}^{x} \frac{u}{D} f(u) u^{\alpha-1} d u \\
& =\frac{\alpha \beta^{\alpha}}{D}-\frac{x \alpha \beta^{\alpha+1}}{D^{2}}+\frac{\alpha \beta^{\alpha}(2 \log x-\log D)}{D}
\end{aligned}
$$

Similarly, we obtain $b_{5}(x)$ as follows:

$$
\begin{aligned}
b_{5}(x) & =\int_{\beta}^{\frac{D}{x}} \frac{x u}{D} f(u) u^{\alpha} d u+\int_{\frac{D}{x}}^{x} f(u) u^{\alpha} d u \\
& =\frac{x \alpha \beta^{\alpha}}{D}\left(\frac{D}{x}-\beta\right)+\alpha \beta^{\alpha}(2 \log x-\log D) \\
& =\alpha \beta^{\alpha}(2 \log x+1-\log D)-\frac{x \alpha \beta^{\alpha+1}}{D} .
\end{aligned}
$$

The derivations of $b_{6}(x)$ and $b_{7}(x)$ are similar to that of $b_{1}(x)$ as follows:

$$
b_{6}(x)=\int_{x}^{\frac{D}{\beta}} f(u) d u=\frac{\beta^{2 \alpha}}{D^{\alpha}}-\frac{\beta^{\alpha}}{x^{\alpha}}
$$

and

$$
b_{7}(x)=\int_{x}^{\frac{D}{\beta}} f(u) u^{\alpha-1} d u=\alpha \beta^{\alpha-1}-\frac{\alpha \beta^{\alpha}}{x} .
$$

2) Deriving $\phi_{i}(x)$ : Now, we are ready to derive $\phi(x)$ with the results of $b_{1}(x)-b_{7}(x)$ and start with $\phi_{1}(x)$ in what follows. Substituting $b_{1}(x)$ and $b_{2}(x)$ in (158)-(159) into $\phi_{11}(x)$ in (115), it follows that:

$$
\begin{aligned}
\phi_{11}(x)= & \frac{x \alpha \beta^{\alpha}}{D^{\alpha-1}(2-\alpha)}\left(-\frac{\alpha \beta^{\alpha+1}}{D^{2}}+\frac{\alpha \beta^{\alpha}}{x D}\right) \\
& +\frac{x \alpha \beta^{2}}{D(2-\alpha)}\left(\frac{\alpha \beta^{2 \alpha-1}}{D^{\alpha}(1-\alpha)}-\frac{\alpha \beta^{\alpha}}{D(1-\alpha)} x^{1-\alpha}\right) \\
= & -\frac{x \alpha^{2} \beta^{2 \alpha+1}}{D^{\alpha+1}(2-\alpha)}+\frac{\alpha^{2} \beta^{2 \alpha}}{D^{\alpha}(2-\alpha)} \\
& +\frac{x \alpha^{2} \beta^{2 \alpha+1}}{D^{\alpha+1}(2-\alpha)(1-\alpha)}-\frac{x^{2-\alpha} \alpha^{2} \beta^{\alpha+2}}{D^{2}(2-\alpha)(1-\alpha)} .
\end{aligned}
$$

Combining (161)-(162) with (121), we obtain $\phi_{12}(x)$ as follows:

$$
\begin{aligned}
\phi_{12}(x)= & \left(\frac{-x^{\alpha-1} \alpha \beta^{\alpha}}{D^{\alpha-1}(2-\alpha)(1-\alpha)}-\frac{x \alpha \beta^{2}}{D(2-\alpha)}\right) \\
\times & \left(-\frac{x^{\alpha-1} \alpha \beta^{\alpha}}{D^{\alpha}(2-\alpha)(1-\alpha)}-\frac{x \alpha \beta^{2}}{D^{2}(2-\alpha)}\right. \\
& \left.+\frac{x^{1-\alpha} \alpha \beta^{\alpha}}{D(1-\alpha)}\right)+\frac{\alpha \beta^{\alpha}}{D^{\alpha-1}(1-\alpha)}\left(\frac{\alpha \beta^{\alpha}}{D}\right. \\
& \left.-\frac{x \alpha \beta^{\alpha+1}}{D^{2}}+\frac{\alpha \beta^{\alpha}(2 \log x-\log D)}{D}\right) .
\end{aligned}
$$

Further simplifying $\phi_{12}(x)$ establishes that:

$$
\begin{aligned}
\phi_{12}(x)= & \frac{x^{2 \alpha-2} \alpha \beta^{2 \alpha}}{D^{2 \alpha-1}(2-\alpha)^{2}(1-\alpha)^{2}}+\frac{x^{\alpha} \alpha^{2} \beta^{\alpha+2}}{D^{\alpha+1}(2-\alpha)^{2}(1-\alpha)} \\
& -\frac{\alpha^{2} \beta^{2 \alpha}}{D^{\alpha}(2-\alpha)(1-\alpha)^{2}}+\frac{x^{\alpha} \alpha^{2} \beta^{\alpha+2}}{D^{\alpha+1}(2-\alpha)^{2}(1-\alpha)} \\
& +\frac{x^{2} \alpha^{2} \beta^{4}}{D^{3}(2-\alpha)^{2}}-\frac{x^{2-\alpha} \alpha^{2} \beta^{\alpha+2}}{D^{2}(2-\alpha)(1-\alpha)}+\frac{\alpha^{2} \beta^{2 \alpha}}{D^{\alpha}(1-\alpha)} \\
& -\frac{x \alpha^{2} \beta^{2 \alpha+1}}{D^{\alpha+1}(1-\alpha)}-\frac{\alpha^{2} \beta^{2 \alpha}(\log D-2 \log x)}{D^{\alpha}(1-\alpha)} .
\end{aligned}
$$

Next, we examine $\phi_{2}(x)$. It follows from (163) that $\phi_{21}(x)$ is given by:

$$
\begin{aligned}
\phi_{21}(x) & =\frac{\beta^{\alpha}}{D^{\alpha}}\left(\alpha \beta^{\alpha}(2 \log x+1-\log D)-\frac{x \alpha \beta^{\alpha+1}}{D}\right) \\
& =\frac{\alpha \beta^{2 \alpha}(2 \log x+1-\log D)}{D^{\alpha}}-\frac{x \alpha \beta^{2 \alpha+1}}{D^{\alpha+1}} .
\end{aligned}
$$

Replacing $b_{6}(x)$ and $b_{7}(x)$ with the results of (164)-(165) in $\phi_{22}(x)$, we establish that:

$$
\begin{aligned}
\phi_{22}(x) & =\frac{\alpha \beta^{\alpha}}{D^{\alpha}(1-\alpha)}\left(\frac{x^{\alpha} \beta^{2 \alpha}}{D^{\alpha}}-\beta^{\alpha}-\frac{x \alpha \beta^{\alpha+1}}{D}+\alpha \beta^{\alpha}\right) \\
& =\frac{x^{\alpha} \alpha \beta^{3 \alpha}}{D^{2 \alpha}(1-\alpha)}-\frac{x \alpha^{2} \beta^{2 \alpha+1}}{D^{\alpha+1}(1-\alpha)}-\frac{\alpha \beta^{2 \alpha}}{D^{\alpha}} .
\end{aligned}
$$

Substituting (164) into $\phi_{23}(x)$ in (135) gives:

$$
\phi_{23}(x)=\frac{x^{\alpha} \beta^{\alpha}}{D^{\alpha}}\left(\frac{\beta^{2 \alpha}}{D^{\alpha}}-\frac{\beta^{\alpha}}{x^{\alpha}}\right)=\frac{x^{\alpha} \beta^{3 \alpha}}{D^{2 \alpha}}-\frac{\beta^{2 \alpha}}{D^{\alpha}} .
$$

The result of $\phi_{3}(x)$ for $x \in\left[\sqrt{D}, \frac{D}{\beta}\right)$ is the same as for $x \in$ $[\beta, \sqrt{D})$ and is given in (154).

3) Identifying Significant Items: From (166)-(171), it follows that the leading terms in $\phi_{1}(x), \phi_{2}(x)$, and $\phi_{3}(x)$ are given by:

$$
\begin{gathered}
\theta_{1}(x)=\frac{2 x^{\alpha} \alpha^{2} \beta^{\alpha+2}}{D^{\alpha+1}(2-\alpha)^{2}(1-\alpha)}, \quad \theta_{2}(x)=\frac{x \alpha^{2} \beta^{2 \alpha-1}}{D^{\alpha}(\alpha-1)}, \\
\theta_{3}(x)=\frac{x \alpha^{2} \beta^{2 \alpha+1}}{D^{\alpha+1}(\alpha-1)} .
\end{gathered}
$$

From the fact that $\alpha<2$ and $x \geq \sqrt{D}$, it is straightforward to verify that only $\theta_{2}(x)$ is dominant in $\theta(x)$ and we get:

$$
\theta(x)=\frac{x \alpha^{2} \beta^{2 \alpha-1}}{D^{\alpha}(\alpha-1)}=\Theta\left(x n^{-\alpha}\right),
$$

from which the second line in (38) holds.

\section{Assembling $\phi(x)$ for $x \in\left[\frac{D}{\beta}, \infty\right)$ and $\alpha \in(1,2)$}

Last, consider the case of $x>\frac{D}{\beta}$, from which we have $\pi(x, u)=1$ and $\pi(x, v)=1$. Therefore, we replace all appearances of $\pi(x, u)$ and $\pi(x, v)$ in $\phi(x)$ with 1 and directly derive the double integral. For $\phi_{1}(x)$, its inner integral has 
range $\left[\beta, \frac{D}{u}\right]$, which gives $\pi(u, v)=\frac{u v}{D}$. It follows that $\phi_{1}(x)$ is given by:

$$
\begin{aligned}
\phi_{1}(x)= & \int_{\beta}^{\frac{D}{\beta}} \int_{\beta}^{\frac{D}{u}} \frac{u v}{D} f(u) f(v) d u d v \\
= & \int_{\beta}^{\frac{D}{\beta}} \frac{\alpha \beta^{\alpha}}{D(1-\alpha)} u f(u)\left(\left(\frac{D}{u}\right)^{1-\alpha}-\beta^{1-\alpha}\right) d u \\
= & \frac{\alpha^{2} \beta^{2 \alpha}(\log D-2 \log \beta)}{D^{\alpha}(1-\alpha)}-\frac{\alpha^{2} \beta^{2 \alpha}}{D^{\alpha}(1-\alpha)^{2}} \\
& +\frac{\alpha^{2} \beta^{2}}{D(1-\alpha)}
\end{aligned}
$$

For $\phi_{2}(x)$, its inner integral has range $\left[\frac{D}{u}, \infty\right)$, which gives $\pi(u, v)=1$. It follows that $\phi_{2}(x)$ is given by:

$$
\begin{aligned}
\phi_{2}(x) & =\int_{\beta}^{\frac{D}{\beta}} \int_{\frac{D}{u}}^{\infty} f(u) f(v) d v d u=\frac{\beta^{\alpha}}{D^{\alpha}} \int_{\beta}^{\frac{D}{\beta}} u^{\alpha} f(u) d u \\
& =\frac{\alpha \beta^{2 \alpha}(\log D-2 \log \beta)}{D^{\alpha}}
\end{aligned}
$$

For $\phi_{3}(x)$, since the outside integral has $u>\frac{D}{\beta}$, we thus obtain $\pi(u, v)=1$ for any $v$, which leads to:

$$
\begin{aligned}
\phi_{3}(x) & =\int_{\frac{D}{\beta}}^{\infty} \int_{\beta}^{\infty} f(u) f(v) d v d u=\frac{\beta^{2 \alpha}}{D^{\alpha}} \int_{\beta}^{\infty} f(v) d v \\
& =\frac{\beta^{2 \alpha}}{D^{\alpha}} .
\end{aligned}
$$

Combining the fact that $\alpha>1$, it thus is easy to verify that the leading terms in $\phi_{1}(x), \phi_{2}(x)$, and $\phi_{3}(x)$ are given by:

$$
\begin{gathered}
\theta_{1}(x)=\frac{\alpha^{2} \beta^{2}}{D(1-\alpha)}, \quad \theta_{2}(x)=\frac{\alpha \beta^{2 \alpha} \log D}{D^{\alpha}}, \\
\theta_{3}(x)=\frac{\beta^{2 \alpha}}{D^{\alpha}} .
\end{gathered}
$$

Since $\alpha>1$, it thus follows that $\theta_{1}(x)$ is dominant and:

$$
\theta(x)=\frac{\alpha^{2} \beta^{2}}{D(1-\alpha)}=\Theta\left(n^{-1}\right),
$$

which leads to the third line in (38).

\section{APPENDIX II \\ PROOF OF LEMMA 6}

Proof: Following the same reasoning as in the proof of Lemma 5 , we identify the leading terms in $\phi_{i}(x)$ for various ranges of $x$.

\section{A. Decomposing $\phi(x)$}

Note that all expressions for $\phi_{2}(x)$ and $\phi_{3}(x)$ are the same as in $\alpha<2$. We only need to derive $\phi_{1}(x)$ for $\alpha=2$. For $\phi_{11}(x)$, we obtain:

$$
\begin{aligned}
\phi_{11}(x) & =\int_{x}^{\frac{D}{\beta}} g(u) \frac{x}{D} \int_{\beta}^{\frac{D}{u}} \alpha \beta^{\alpha} v^{1-\alpha} d v d u \\
& =\int_{x}^{\frac{D}{\beta}} g(u) \frac{x \alpha \beta^{\alpha}}{D}\left(\log \frac{D}{\beta}-\log u\right) d u .
\end{aligned}
$$

If we define $b_{8}(x)$ to be:

$$
b_{8}(x)=\int_{x}^{\frac{D}{\beta}} g(u) \log u d u
$$

then we reduce $\phi_{11}(x)$ to:

$$
\phi_{11}(x)=\frac{x \alpha \beta^{\alpha}(\log D-\log \beta)}{D} b_{2}(x)-\frac{x \alpha \beta^{\alpha}}{D} b_{8}(x),
$$

where $b_{2}(x)$ is given in (114).

Similarly, for $\phi_{12}(x)$, it follows that:

$$
\begin{aligned}
\phi_{12}(x)= & \int_{\beta}^{x} g(u) \frac{x \alpha \beta^{\alpha}}{D} \log \frac{D}{x \beta} d u \\
& +\int_{\beta}^{x} g(u) \frac{\alpha \beta^{\alpha}}{1-\alpha}\left(\left(\frac{D}{u}\right)^{1-\alpha}-\left(\frac{D}{x}\right)^{1-\alpha}\right) d u \\
= & \left(\frac{x \alpha \beta^{\alpha}}{D} \log \frac{D}{x \beta}-\frac{x^{\alpha-1} \alpha \beta^{\alpha}}{D^{\alpha-1}(1-\alpha)}\right) \int_{\beta}^{x} g(u) d u \\
& +\frac{\alpha \beta^{\alpha}}{D^{\alpha-1}(1-\alpha)} \int_{\beta}^{x} g(u) u^{\alpha-1} d u .
\end{aligned}
$$

Using the definitions of $b_{3}(x)$ and $b_{4}(x)$ in (120), we can simplify $\phi_{12}(x)$ as follows:

$$
\begin{aligned}
\phi_{12}(x)= & \left(\frac{x \alpha \beta^{\alpha}}{D} \frac{D}{x \beta}-\frac{x^{\alpha-1} \alpha \beta^{\alpha}}{D^{\alpha-1}(1-\alpha)}\right) b_{3}(x) \\
& +\frac{\alpha \beta^{\alpha}}{D^{\alpha-1}(1-\alpha)} b_{4}(x) .
\end{aligned}
$$

Now, we proceed to derive $\phi(x)$ by solving $b_{1}(x)-b_{8}(x)$ for $x \in[\beta, \sqrt{D})$ and $\alpha=2$.

\section{B. Assembling $\phi(x)$ for $x \in[\beta, \sqrt{D})$ and $\alpha=2$}

Notice that $b_{1}(x), b_{4}(x), b_{5}(x), b_{6}(x)$, and $b_{7}(x)$ are the same as for $\alpha<2$ and $x \in[\beta, \sqrt{D})$. Next, we only need to derive $b_{2}(x), b_{3}(x)$, and $b_{8}(x)$ for $\alpha=2$. It is straightforward to obtain $b_{2}(x)$ and $b_{3}(x)$ as follows:

$$
\begin{aligned}
b_{2}(x)= & \frac{x \alpha \beta^{\alpha}}{D^{2}} \int_{x}^{\frac{D}{x}} u^{1-\alpha} d u+\frac{\alpha \beta^{\alpha}}{D} \int_{\frac{D}{x}}^{\frac{D}{\beta}} u^{-\alpha} d u \\
= & \frac{x \alpha \beta^{\alpha}}{D^{2}}(\log D-2 \log x)+\frac{\alpha \beta^{2 \alpha-1}}{D^{\alpha}(1-\alpha)} \\
& -\frac{x^{\alpha-1} \alpha \beta^{\alpha}}{D^{\alpha}(1-\alpha)}
\end{aligned}
$$

and

$$
b_{3}(x)=\frac{x \alpha \beta^{\alpha}}{D^{2}} \int_{\beta}^{x} u^{1-\alpha} d u=\frac{x \alpha \beta^{\alpha}}{D^{2}}(\log x-\log \beta) .
$$


As for $b_{8}(x)$, we can split the integral on $\left[\beta, \frac{D}{\beta}\right]$ into two parts, $\left[\beta, \frac{D}{x}\right]$ and $\left[\frac{D}{x}, \frac{D}{\beta}\right]$, where $\pi(x, u)=\frac{x u}{D}$ and 1 , respectively.

$$
\begin{aligned}
b_{8}(x)= & \int_{x}^{\frac{D}{\beta}} g(u) \log u d u \\
= & \int_{x}^{\frac{D}{x}} \frac{u}{D} \frac{u x}{D} f(u) \log u d u+\int_{\frac{D}{x}}^{\frac{D}{\beta}} \frac{u}{D} f(u) \log u d u \\
= & \frac{x \alpha \beta^{\alpha}}{2 D^{2}}\left(\log ^{2} \frac{D}{x}-\log ^{2} x\right)+\frac{\alpha \beta^{\alpha}}{D(1-\alpha)} \\
& \times\left(\left(\frac{D}{\beta}\right)^{1-\alpha} \log \frac{D}{\beta}-\left(\frac{D}{x}\right)^{1-\alpha} \log \frac{D}{x}\right) \\
& \quad-\frac{\alpha \beta^{\alpha}}{D(1-\alpha)^{2}}\left(\left(\frac{D}{\beta}\right)^{1-\alpha}-\left(\frac{D}{x}\right)^{1-\alpha}\right) .
\end{aligned}
$$

Simplifying $b_{8}(x)$ establishing the following equality:

$$
\begin{aligned}
b_{8}(x)= & \frac{x \alpha \beta^{\alpha} \log D(\log D-2 \log x)}{2 D^{2}}+\frac{\alpha \beta^{2 \alpha-1}}{D^{\alpha}(1-\alpha)} \log \frac{D}{\beta} \\
& -\frac{x^{\alpha-1} \alpha \beta^{\alpha}}{D^{\alpha}(1-\alpha)} \log \frac{D}{x}-\frac{\alpha \beta^{2 \alpha-1}+x^{\alpha-1} \alpha \beta^{\alpha}}{D^{\alpha}(1-\alpha)^{2}} .
\end{aligned}
$$

By substituting (184) and (187) into $\phi_{11}(x)$ in (181), we get:

$$
\begin{aligned}
\phi_{11}(x)= & \frac{x \alpha \beta^{\alpha} \log \frac{D}{\beta}}{D}\left(\frac{x \alpha \beta^{\alpha}}{D^{2}} \log \frac{D}{x^{2}}\right. \\
& \left.+\frac{\alpha \beta^{2 \alpha-1}}{D^{\alpha}(1-\alpha)}-\frac{x^{\alpha-1} \alpha \beta^{\alpha}}{D^{\alpha}(1-\alpha)}\right) \\
- & \frac{x \alpha \beta^{\alpha}}{D}\left(\frac{x \alpha \beta^{\alpha} \log D}{2 D^{2}} \log \frac{D}{x^{2}}\right. \\
& +\frac{\alpha \beta^{2 \alpha-1}}{D^{\alpha}(1-\alpha)} \log \frac{D}{\beta}-\frac{x^{\alpha-1} \alpha \beta^{\alpha}}{D^{\alpha}(1-\alpha)} \log \frac{D}{x} \\
& \left.-\frac{\alpha \beta^{2 \alpha-1}}{D^{\alpha}(1-\alpha)^{2}}-\frac{x^{\alpha-1} \alpha \beta^{\alpha}}{D^{\alpha}(1-\alpha)^{2}}\right) .
\end{aligned}
$$

Further simplifying $\phi_{11}(x)$, we obtain that the next formula holds:

$$
\begin{aligned}
\phi_{11}(x)= & \frac{x^{2} \alpha^{2} \beta^{2 \alpha}}{D^{3}} \log \frac{D}{x} \log \frac{D}{\beta}+\frac{x \alpha^{2} \beta^{3 \alpha-1}}{D^{\alpha+1}(1-\alpha)} \log \frac{D}{\beta} \\
& -\frac{x^{\alpha} \alpha^{2} \beta 2 \alpha}{D^{\alpha+1}(1-\alpha)} \log \frac{D}{\beta}-\frac{x^{2} \alpha^{2} \beta^{2 \alpha} \log D}{2 D^{3}} \log \frac{D}{x^{2}} \\
& -\frac{x \alpha^{2} \beta^{3 \alpha-1}}{D^{\alpha+1}(1-\alpha)} \log \frac{D}{\beta}+\frac{x^{\alpha} \alpha^{2} \beta^{2 \alpha}}{D^{\alpha+1}(1-\alpha)} \log \frac{D}{x} \\
& +\frac{x \alpha^{2} \beta^{3 \alpha-1}}{D^{\alpha+1}(1-\alpha)^{2}}-\frac{x^{\alpha} \alpha^{2} \beta^{2 \alpha}}{D^{\alpha+1}(1-\alpha)^{2}} .
\end{aligned}
$$

Similarly, from (185) and (142), we have:

$$
\begin{aligned}
\phi_{12}(x)= & \left(\frac{x \alpha \beta^{\alpha}}{D} \log \frac{D}{x \beta}-\frac{x^{\alpha-1} \alpha \beta^{\alpha}}{D^{\alpha-1}(1-\alpha)}\right) \frac{x \alpha \beta^{\alpha}}{D^{2}} \log \frac{x}{\beta} \\
& +\frac{\alpha \beta^{\alpha}}{D^{\alpha-1}(1-\alpha)}\left(\frac{x^{2} \alpha \beta^{\alpha}}{D^{2}}-\frac{x \alpha \beta^{\alpha+1}}{D^{2}}\right)
\end{aligned}
$$

which establishes:

$$
\begin{aligned}
\phi_{12}(x)= & \frac{x^{2} \alpha^{2} \beta^{2 \alpha}}{D^{3}} \log \frac{D x}{\beta} \log \frac{x}{\beta}-\frac{x^{\alpha} \alpha^{2} \beta^{2 \alpha}}{D^{\alpha+1}(1-\alpha)} \log \frac{x}{\beta} \\
& +\frac{x^{2} \alpha^{2} \beta^{2 \alpha}}{D^{\alpha+1}(1-\alpha)}-\frac{x \alpha^{2} \beta^{2 \alpha+1}}{D^{\alpha+1}(1-\alpha)}
\end{aligned}
$$

Therefore, the leading term $\theta_{1}(x)$ in $\phi_{1}(x)$ is given by:

$$
\theta_{1}(x)=\frac{x^{2} \alpha^{2} \beta^{2 \alpha} \log ^{2} D}{D^{3}} .
$$

Notice that $\phi_{2}(x)$ and $\phi_{3}(x)$ are the same as for $\alpha<2$. Therefore, it follows from (153) and (154) that the leading terms in $\phi_{2}(x)$ and $\phi_{3}(x)$ are as follows:

$$
\theta_{2}(x)=\frac{x^{2} \alpha^{2} \beta^{2 \alpha} \log D}{D^{3}(\alpha-1)}, \quad \theta_{3}(x)=\frac{x \alpha^{2} \beta^{2 \alpha+1}}{D^{3}(\alpha-1)} .
$$

Therefore, we have the leading term in $\phi(x)$ :

$$
\theta(x)=\frac{x^{2} \alpha^{2} \beta^{2 \alpha} \log ^{2} D}{D^{3}}=\Theta\left(x^{2} n^{-3} \log ^{2} n\right),
$$

which gives the first line of (39).

\section{Assembling $\phi(x)$ for $x \in\left[\sqrt{D}, \frac{D}{\beta}\right)$ and $\alpha=2$}

Next, consider the case of $x \in\left[\sqrt{D}, \frac{D}{\beta}\right)$, where all $b_{i}(x)$ except $b_{3}(x)$ and $b_{8}(x)$ are the same as for $\alpha<2$. Therefore, we only need to recompute $b_{3}(x)$ and $b_{8}(x)$ for $\alpha=2$. For $b_{3}(x)$, we split the integral range $[\beta, x]$ into two parts: $\left[\beta, \frac{D}{x}\right]$ and $\left[\frac{D}{x}, x\right]$, where $\pi(x, u)=\frac{x u}{D}$ and 1 , respectively. It follows that:

$$
\begin{aligned}
b_{3}(x) & =\int_{\beta}^{x} \frac{u}{D} \pi(x, u) f(u) d u \\
& =\int_{\beta}^{\frac{D}{x}} \frac{u}{D} \frac{x u}{D} f(u) d u+\int_{\frac{D}{x}}^{x} \frac{u}{D} f(u) d u \\
& =\frac{x \alpha \beta^{\alpha}}{D^{2}} \int_{\beta}^{\frac{D}{x}} u^{1-\alpha} d u+\frac{\alpha \beta^{\alpha}}{D} \int_{\frac{D}{x}}^{x} u^{-\alpha} d u .
\end{aligned}
$$

We simply $b_{3}(x)$ to obtain the following equation:

$$
\begin{aligned}
b_{3}(x) & =\frac{x \alpha \beta^{\alpha}}{D^{2}} \log \frac{D}{x \beta}+\frac{\alpha \beta^{\alpha}}{D(1-\alpha)}\left(x^{1-\alpha}-\left(\frac{D}{x}\right)^{1-\alpha}\right) \\
& =\frac{x \alpha \beta^{\alpha}}{D^{2}} \log \frac{D}{x \beta}+\frac{x^{1-\alpha} \alpha \beta^{\alpha}}{D(1-\alpha)}-\frac{x^{\alpha-1} \alpha \beta^{\alpha}}{D^{\alpha}(1-\alpha)} .
\end{aligned}
$$

For $b_{8}(x)$, it follows that:

$$
\begin{aligned}
b_{8}(x) & =\int_{x}^{\frac{D}{\beta}} g(u) \log u d u=\int_{x}^{\frac{D}{\beta}} \frac{u}{D} f(u) \log u d u \\
& =\frac{\alpha \beta^{\alpha}}{D} \int_{x}^{\frac{D}{\beta}} u^{-2} \log u d u .
\end{aligned}
$$


Integrating by part the right-hand side of (197), we obtain:

$$
\begin{aligned}
b_{8}(x)= & \frac{\alpha \beta^{\alpha}}{D(1-\alpha)}\left(\left(\frac{D}{\beta}\right)^{1-\alpha} \log \frac{D}{\beta}-x^{1-\alpha} \log x\right) \\
& -\frac{\alpha \beta^{\alpha}}{D(1-\alpha)^{2}}\left(\left(\frac{D}{\beta}\right)^{1-\alpha}-x^{1-\alpha}\right) \\
= & \frac{\alpha \beta^{2 \alpha-1}}{D^{\alpha}(1-\alpha)} \log \frac{D}{\beta}-\frac{\alpha \beta^{\alpha} x^{1-\alpha} \log x}{D(1-\alpha)} \\
& -\frac{\alpha \beta^{2 \alpha-1}}{D^{\alpha}(1-\alpha)^{2}}+\frac{\alpha \beta^{\alpha} x^{1-\alpha}}{D(1-\alpha)^{2}} .
\end{aligned}
$$

Now, we are ready to derive $\phi_{i}(x)$. Substituting (158) and (198) into $\phi_{11}(x)$ in (181) results in:

$$
\begin{aligned}
\phi_{11}(x)= & \frac{x \alpha \beta^{\alpha} \log \frac{D}{\beta}}{D}\left(\frac{\alpha \beta^{2 \alpha-1}}{D^{\alpha}(1-\alpha)}-\frac{\alpha \beta^{\alpha} x^{1-\alpha}}{D(1-\alpha)}\right) \\
- & \frac{x \alpha \beta^{\alpha}}{D}\left(\frac{\alpha \beta^{2 \alpha-1}}{D^{\alpha}(1-\alpha)} \log \frac{D}{\beta}-\frac{\alpha \beta^{\alpha} x^{1-\alpha} \log x}{D(1-\alpha)}\right. \\
& \left.-\frac{\alpha \beta^{2 \alpha-1}}{D^{\alpha}(1-\alpha)^{2}}+\frac{\alpha \beta^{\alpha} x^{1-\alpha}}{D(1-\alpha)^{2}}\right)
\end{aligned}
$$

which leads to the following formula:

$$
\begin{aligned}
\phi_{11}(x)= & \frac{x \alpha^{2} \beta^{3 \alpha-1}}{D^{\alpha+1}(1-\alpha)} \log \frac{D}{\beta}-\frac{x^{2-\alpha} \alpha^{2} \beta^{2 \alpha}}{D^{2}(1-\alpha)} \log \frac{D}{\beta} \\
& -\frac{x \alpha^{2} \beta^{3 \alpha-1}}{D^{\alpha+1}(1-\alpha)} \log \frac{D}{\beta}+\frac{x^{2-\alpha} \alpha^{2} \beta^{2 \alpha} \log x}{D^{2}(1-\alpha)} \\
& +\frac{x \alpha^{2} \beta^{3 \alpha-1}}{D^{\alpha+1}(1-\alpha)^{2}}-\frac{x^{2-\alpha} \alpha^{2} \beta^{2 \alpha}}{D^{2}(1-\alpha)^{2}}
\end{aligned}
$$

Combining with (162) and (196), $\phi_{12}(x)$ in (183) reduces to:

$$
\begin{aligned}
\phi_{12}(x)= & \left(\frac{x \alpha \beta^{\alpha}}{D} \log \frac{D}{x \beta}-\frac{x^{\alpha-1} \alpha \beta^{\alpha}}{D^{\alpha-1}(1-\alpha)}\right) \\
& \times\left(\frac{x \alpha \beta^{\alpha}}{D^{2}} \log \frac{D}{x \beta}+\frac{x^{1-\alpha} \alpha \beta^{\alpha}}{D(1-\alpha)}-\frac{x^{\alpha-1} \alpha \beta^{\alpha}}{D^{\alpha}(1-\alpha)}\right) \\
+ & \frac{\alpha \beta^{\alpha}}{D^{\alpha-1}(1-\alpha)}\left(\frac{\alpha \beta^{\alpha}}{D}-\frac{x \alpha \beta^{\alpha+1}}{D^{2}}\right. \\
& \left.-\frac{\alpha \beta^{\alpha}}{D} \log \frac{D}{x^{2}}\right) .
\end{aligned}
$$

Then, we reduce $\phi_{12}(x)$ to become:

$$
\begin{aligned}
\phi_{12}(x)= & \frac{x^{2} \alpha^{2} \beta^{2 \alpha}}{D^{3}}\left(\log \frac{D}{x \beta}\right)^{2}+\frac{x^{2-\alpha} \alpha^{2} \beta 2 \alpha}{D^{2}(1-\alpha)} \log \frac{D}{x \beta} \\
& -\frac{2 x^{\alpha} \alpha^{2} \beta^{2 \alpha}}{D^{\alpha+1}(1-\alpha)} \log \frac{D}{x \beta}-\frac{\alpha^{2} \beta^{2 \alpha}}{D^{\alpha}(1-\alpha)^{2}} \\
& +\frac{x^{2 \alpha-1} \alpha^{2} \beta^{2 \alpha}}{D^{2 \alpha-1}(1-\alpha)^{2}}-\frac{\alpha^{2} \beta^{2 \alpha}}{D^{\alpha}(1-\alpha)} \\
& +\frac{x \alpha^{2} \beta^{2 \alpha+1}}{D^{\alpha+1}(1-\alpha)}-\frac{\alpha^{2} \beta^{2 \alpha}}{D^{\alpha}(1-\alpha)} \log \frac{D}{x^{2}} .
\end{aligned}
$$

Considering $\alpha=2$, it thus follows that the leading term in $\phi_{1}(x)$ is given by:

$$
\theta_{1}(x)=\frac{x^{2 \alpha-1} \alpha^{2} \beta^{2 \alpha}}{D^{2 \alpha-1}(1-\alpha)^{2}} .
$$

Combining with (169)-(171), we have the leading term in $\phi_{2}(x)$ is:

$$
\theta_{2}(x)=\frac{x \alpha^{2} \beta^{2 \alpha-1}}{D^{\alpha}(\alpha-1)} .
$$

Notice that $\phi_{3}(x)$ is the same as for $\alpha<2$ and $x \in[\beta, \sqrt{D})$ in (154). It follows that the leading term in $\phi_{3}(x)$ is:

$$
\theta_{3}(x)=\frac{x \alpha^{2} \beta^{2 \alpha+1}}{D^{\alpha+1}(\alpha-1)} .
$$

Since $\theta_{1}(x)$ is dominant, we thus are able to identify the leading term in $\phi(x)$ :

$$
\theta(x)=\frac{x^{2 \alpha-1} \alpha^{2} \beta^{2 \alpha}}{D^{2 \alpha-1}(1-\alpha)^{2}}=\Theta\left(x^{3} n^{-3}\right),
$$

which establishes the second line of (39).

\section{Assembling $\phi(x)$ for $x \in\left[\frac{D}{\beta}, \infty\right)$ and $\alpha=2$}

For $x \in\left[\frac{D}{\beta}, \infty\right)$ with $\alpha=2$, we obtain that $\phi(x)$ is the same as in the already-covered case $1<\alpha<2$. Reusing the results of Lemma 5, we have:

$$
\theta(x)=\Theta\left(n^{-1}\right),
$$

which proves the third line of (39).

\section{APPENDIX III}

\section{PROOF OF LEMMA 5}

Proof: As before, we start with $x \in[\beta, \sqrt{D})$ and obtain that:

$$
\begin{gathered}
\theta_{1}(x)=\frac{x^{2} \alpha^{2} \beta^{4}}{D^{3}(2-\alpha)^{2}}, \quad \theta_{2}(x)=\frac{x^{2} \alpha^{2} \beta^{2 \alpha} \log D}{D^{\alpha+1}(\alpha-1)} \\
\theta_{3}(x)=\frac{x \alpha^{2} \beta^{2 \alpha+1}}{D^{\alpha+1}(\alpha-1)},
\end{gathered}
$$

Since $\alpha>2$, it follows that $\theta_{1}(x)$ is dominant and $\theta(x)=$ $\Theta\left(x^{2} n^{-3}\right)$.

For $x \in\left[\sqrt{D}, \frac{D}{\beta}\right)$, the leading terms $\theta_{i}(x)$ are:

$$
\begin{gathered}
\theta_{1}(x)=\frac{x^{2} \alpha^{2} \beta^{4}}{D^{3}(2-\alpha)^{2}}, \quad \theta_{2}(x)=\frac{2 \alpha \beta^{2 \alpha} \log x}{D^{\alpha}} \\
\theta_{3}(x)=\frac{x \alpha^{2} \beta^{2 \alpha+1}}{D^{\alpha+1}(\alpha-1)},
\end{gathered}
$$

Since $x^{2} \geq D$ and $\alpha>2$, it is easy to verify that $\theta_{1}(x)$ is asymptotically larger than both $\theta_{2}(x)$ and $\theta_{3}(x)$, and therefore $\theta(x)=\Theta\left(x^{2} n^{-3}\right)$.

Finally, for $x \in\left[\frac{D}{\beta}, \infty\right)$, we again obtain $\theta(x)=\Theta\left(n^{-1}\right)$ using straightforward integration. Combining these results, we establish (40). 